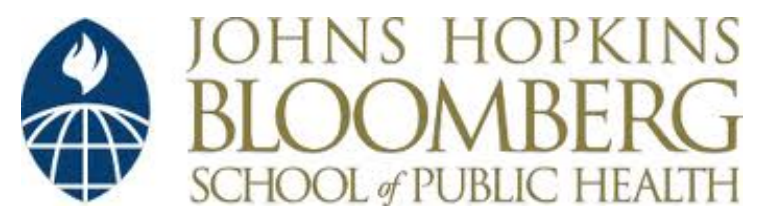

Johns Hopkins University, Dept. of Biostatistics Working Papers

$11-1-2013$

\title{
Joint Estimation of Multiple Graphical Models from High Dimensional Time Series
}

Huitong Qiu

Johns Hopkins University, hqiu@jhsph.edu

Fang Han

Johns Hopkins University, fhan@jhsph.edu

Han Liu

Princeton University, hanliu@princeton.edu

Brian Caffo

Johns Hopkins University, bcaffo@jhsph.edu

\section{Suggested Citation}

Qiu, Huitong; Han, Fang; Liu, Han; and Caffo, Brian, "Joint Estimation of Multiple Graphical Models from High Dimensional Time Series" (November 2013). Johns Hopkins University, Dept. of Biostatistics Working Papers. Working Paper 259.

http://biostats.bepress.com/jhubiostat/paper259

This working paper is hosted by The Berkeley Electronic Press (bepress) and may not be commercially reproduced without the permission of the copyright holder.

Copyright $(\odot) 2011$ by the authors 


\title{
Joint Estimation of Multiple Graphical Models from High Dimensional Time Series
}

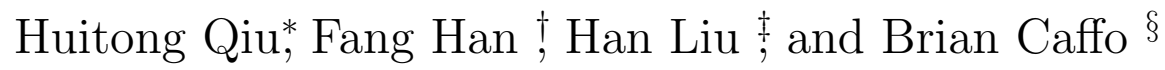

November 1, 2013

\begin{abstract}
In this manuscript the problem of jointly estimating multiple graphical models in high dimensions is considered. It is assumed that the data are collected from $n$ subjects, each of which consists of $m$ non-independent observations. The graphical models of subjects vary, but are assumed to change smoothly corresponding to a measure of the closeness between subjects. A kernel based method for jointly estimating all graphical models is proposed. Theoretically, under a double asymptotic framework, where both $(m, n)$ and the dimension $d$ can increase, the explicit rate of convergence in parameter estimation is provided, thus characterizing the strength one can borrow across different individuals and impact of data dependence on parameter estimation. Empirically, experiments on both synthetic and real resting state functional magnetic resonance imaging (rs-fMRI) data illustrate the effectiveness of the proposed method.
\end{abstract}

Keyword: Graphical model; Conditional independence; High dimensional data; Time series; Rate of convergence.

\footnotetext{
*Department of Biostatistics, Johns Hopkins University, Baltimore, MD 21205, USA; e-mail: hqiu@jhsph.edu

${ }^{\dagger}$ Department of Biostatistics, Johns Hopkins University, Baltimore, MD 21205, USA; e-mail: fhan@jhsph.edu

${ }^{\ddagger}$ Department of Operations Research and Financial Engineering, Princeton University, Princeton, NJ 08544, USA; e-mail: hanliu@princeton.edu

${ }^{\S}$ Department of Biostatistics, Johns Hopkins University, Baltimore, MD 21205, USA; e-mail: bcaffo@gmail.com
} 


\section{Introduction}

Undirected graphical models encode the conditional independence structure among the variables in a random vector, and have been heavily exploited in multivariate data analysis (Lauritzen, 1996). In particular, when $\boldsymbol{X} \sim N_{d}(\mathbf{0}, \boldsymbol{\Sigma})$ is a dimensional random vector, estimating the undirected graphical model is equivalent to estimating the nonzero entries in the inverse covariance matrix $\Theta:=\boldsymbol{\Sigma}^{-1}$ (Dempster, 1972). The undirected graphical model for the Gaussian distribution is thus called the Gaussian graphical model.

There has been much work on estimating a single Gaussian graphical model, G, based on $n$ independent observations. In the low dimensional settings where the dimension, $d$, is fixed, Drton and Perlman (2007) and Drton and Perlman (2008) proposed to estimate $\mathrm{G}$ using multiple testing procedures. In settings where the dimension, $d$, is much larger than the sample size, $n$, Meinshausen and Bühlmann (2006) proposed to estimate Gaussian graphical models by solving a collection of regression problems via the lasso. Yuan and Lin (2007), Banerjee et al. (2008), Friedman et al. (2008), Rothman et al. (2008), and Liu and Luo (2012) proposed to directly estimate $\Theta$ using the $\ell_{1}$ penalty (detailed definition provided later). More recently, Yuan (2010) and Cai et al. (2011) proposed to estimate $\boldsymbol{\Theta}$ via linear programming. The above mentioned estimators are all consistent with regard to both parameter estimation and model selection, even when $d$ is nearly exponential larger than $n$.

These body of work focuses on estimating a single graph based on independent realizations of a common random vector. However, in many applications this simple model cannot hold. For example, the data can be collected from multiple individuals that share the same set of variables, but differ with regard to the structures among variables. This situation is frequently encountered in the areas of brain connectivity network estimation (Friston, 2011). Here brain connectivity networks corresponding to different subjects vary, but are expected to be more similar if the corresponding subjects share many common demographic, health or other covariate features. Under this setting, estimating the graphical models separately for each subject ignores the similarity between the adjacent graphical models. In contrast, estimating one population graphical model based on the data collected from all subjects ignores the differences between graphs and can lead to inconsistent estimates.

There has been a line of research in jointly estimating multiple Gaussian graphical models for independent data. On one hand, Guo et al. (2011) and Danaher et al. (2013) proposed methods via introducing new penalty terms, which encourage the sparsity of both the parameters in each subject and the differences between parameters in different 
subjects. On the other hand, Song et al. (2009a), Song et al. (2009b), Kolar and Xing (2009), Zhou et al. (2010), and Kolar et al. (2010) focused on the independent data with time-varying networks and proposed efficient algorithms for estimating and predicting the networks along the time line.

In this paper a new method for jointly estimating and predicting networks corresponding to multiple subjects is proposed. The method is based on a different model compared to the ones listed above. The motivation of this model arises from resting state fMRI data, where there exist many natural orderings corresponding to measures of health status, demographics, and many other subject-specific covariates. Moreover, the observations of each subject are multiple brain scans with temporal dependence. Accordingly, different from the methods in estimating time varying networks, we need to handle the data where each subject has $m$, instead of one, observations. Different from the methods in Guo et al. (2011) and Danaher et al. (2013), it is assumed that there exists a natural ordering for the subjects, and the parameters of interest vary smoothly corresponding to this ordering. Moreover, the observations are allowed to be non-independent via a temporal dependence structure, which has not been studied in high dimensions until very recently (Loh and Wainwright, 2012; Han and Liu, 2013; Wang et al., 2013).

Exploiting a similar kernel based approach as exploited in Zhou et al. (2010), it is shown that our method can efficiently estimate and predict multiple networks while allowing the data to be non-independent. Theoretically, under a double asymptotic framework, where both $d$ and $(m, n)$ can increase, an explicit rate of convergence in parameter estimation is provided, sharply characterizing the strength one can borrow across different subjects and the impact of data dependence on the convergence rate. Empirically, the effectiveness of the proposed method is illustrated on both synthetic and actual resting state function magnetic resonance imaging (rs-fMRI) data. In detail, comparisons of the proposed approach with several existing methods are conducted under three synthetic patterns of evolving graphs. In addition, the large scale ADHD-200 dataset is exploited to investigate the development of brain connectivity networks over age, as well as the effect of kernel bandwidth on estimation, where interesting scientific results are unveiled.

The rest of the paper is organized as follows. In Section 2, the problem setup is introduced and the proposed method is given. In Section 3, the main theoretical results are provided. In Section 4, the method is applied on both synthetic and rs-fMRI data to illustrate its empirical usefulness. A discussion is provided in the last section, while detailed technical proofs are put in the appendix. 


\section{The Model and Method}

Let $\mathbf{M}=\left(M_{j k}\right) \in \mathbb{R}^{d \times d}$ and $\boldsymbol{v}=\left(v_{1}, \ldots, v_{d}\right)^{T} \in \mathbb{R}^{d}$. We denote $\boldsymbol{v}_{I}$ to be the subvector of $\boldsymbol{v}$ whose entries are indexed by a set $I \subset\{1, \ldots, d\}$. We denote $\mathbf{M}_{I, J}$ to be the submatrix of $\mathbf{M}$ whose rows are indexed by $I$ and columns are indexed by $J$. Let $\mathbf{M}_{I, *}$ to be the submatrix of $\mathbf{M}$ whose rows are indexed by $I, \mathbf{M}_{*, J}$ to be the submatrix of $\mathbf{M}$ whose columns are indexed by $J$. For $0<q<\infty$, define the $\ell_{0}, \ell_{q}$, and $\ell_{\infty}$ vector norms as

$$
\|\boldsymbol{v}\|_{0}=\sum_{j=1}^{d} I\left(v_{j} \neq 0\right), \quad\|\boldsymbol{v}\|_{q}:=\left(\sum_{j=1}^{d}\left|v_{j}\right|^{q}\right)^{1 / q}, \quad \text { and }\|\boldsymbol{v}\|_{\infty}=\max _{1 \leq j \leq d}\left|v_{j}\right|,
$$

where $I(\cdot)$ is the indicator function. For a matrix $\mathbf{M}$, denote the matrix $\ell_{q}$, $\ell_{\max }$, and Frobenius norms to be

$$
\|\mathbf{M}\|_{q}=\max _{\|\boldsymbol{v}\|_{q}=1}\|\mathbf{M} \boldsymbol{v}\|_{q},\|\mathbf{M}\|_{\max }=\max _{j k}\left|M_{j k}\right|, \text { and }\|\mathbf{M}\|_{F}=\left(\sum_{j, k}\left|M_{j k}\right|^{2}\right)^{1 / 2} .
$$

For any two sequences $a_{n}, b_{n} \in \mathbb{R}$, we say that $a_{n} \asymp b_{n}$ if $c b_{n} \leq a_{n} \leq C b_{n}$ for some constants $c, C$.

\section{$2.1 \quad$ Model}

Let $\left\{\boldsymbol{X}^{t}\right\}_{t \in[0,1]}$ be a series of $d$-dimensional random vectors indexed by the label $t$, which can represent any kind of ordering in subjects (e.g., any covariate or confounder of interest transformed to the space $[0,1])$. For any $t \in[0,1]$, assume that $\boldsymbol{X}^{t} \sim N_{d}\{\mathbf{0}, \boldsymbol{\Sigma}(t)\}$. Here $\Sigma(\cdot):[0,1] \rightarrow \mathbb{S}_{+}^{d \times d}$ is a function on $[0,1]$ to the $d$ by $d$ positive definite matrix set, represented as $\mathbb{S}_{+}^{d \times d}$. Let $\boldsymbol{\Omega}(t):=\{\boldsymbol{\Sigma}(t)\}^{-1}$ be the inverse covariance matrix of $\boldsymbol{X}^{t}$ and let $\mathbf{G}(t) \in\{0,1\}^{d \times d}$ represent the conditional independence graph corresponding to $\boldsymbol{X}^{t}$, satisfying that

$$
\{\mathbf{G}(t)\}_{j k}=1 \text { if and only if }\{\boldsymbol{\Omega}(t)\}_{j k} \neq 0 .
$$

Suppose that data points in $t=t_{1}, \ldots, t_{n}$ are observed. Let $\boldsymbol{x}_{i 1}, \ldots, \boldsymbol{x}_{i m} \in \mathbb{R}^{d}$ be $m$ possibly non-independent observations of $\boldsymbol{X}^{t_{i}}$, with a temporal dependence structure among them. In particular, for simplicity, in this manuscript we assume that $\left\{\boldsymbol{x}_{i l}\right\}_{l=1}^{m}$ follows a lag one stationary vector autoregressive model, i.e., satisfying that

$$
\boldsymbol{x}_{i l}=\mathbf{A}\left(t_{i}\right) \boldsymbol{x}_{i(l-1)}+\boldsymbol{\epsilon}_{i l}, \text { for } i=1, \ldots, n, l=2, \ldots, m \text {. }
$$


Here we note that extensions to vector autoregressive models with higher orders are also analyzable by using the same techniques in Han and Liu (2013). But for simplicity in this manuscript we only consider the lag one case. $\mathbf{A}(t) \in \mathbb{R}^{d \times d}$ is referred to as the transition matrix. It is assumed that the Gaussian noise, $\boldsymbol{\epsilon}_{i l} \sim N_{d}\left\{\mathbf{0}, \mathbf{\Psi}\left(t_{i}\right)\right\}$ is independent of $\left\{\boldsymbol{\epsilon}_{i l^{\prime}}\right\}_{l^{\prime} \neq l}$ and $\left\{\boldsymbol{x}_{i l^{\prime}}\right\}_{l^{\prime}=1}^{l-1}$. Both $\mathbf{A}(\cdot)$ and $\boldsymbol{\Psi}(\cdot)$ are considered as functions on $[0,1]$. Due to the stationary property, for any $t \in[0,1]$ we have that

$$
\boldsymbol{\Sigma}(t)=\mathbf{A}(t) \boldsymbol{\Sigma}(t)\{\mathbf{A}(t)\}^{T}+\mathbf{\Psi}(t)
$$

holds. For any $i \neq i^{\prime}$, it is assumed that $\left\{\boldsymbol{x}_{i l}\right\}_{l=1}^{m}$ are independent of $\left\{\boldsymbol{x}_{i^{\prime} l}\right\}_{l=1}^{m}$. For $i=$ $1, \ldots, n$ and $l=1, \ldots, m$, denote $\boldsymbol{x}_{i l}=\left(x_{i l 1}, \ldots, x_{i l d}\right)^{T}$.

As noted in Section 1, the application that motivates this model is to estimate subjectspecific brain networks using resting state fMRI data. As an example, the ADHD data considered in Section 4.3 consist of $n$ subjects with ages ranging in $t_{1}, \ldots, t_{n}$, where for each subject $i$, a list of rs-fMRI images with temporal dependence are available. The vector autoregressive model, as exploited in Equation (2.1), is a common tool in modeling dependence for rs-fMRI data. Consider Harrison et al. (2003), Penny et al. (2005), Rogers et al. (2010), Chen et al. (2011), and Valdés-Sosa et al. (2005), for more details.

\subsection{Method}

We exploite the idea proposed in Zhou et al. (2010) and uses a kernel based estimator to borrow strength for subject specific graph estimation. The proposed approach requires two main steps. In the first step, a smoothed covariance matrix estimate of $\boldsymbol{\Sigma}(t)$, denoted as $\mathbf{S}(t)$, is obtained for any given label $t$. In the second step, $\Omega(t)$ is estimated by plugging the covariance matrix estimate $\mathbf{S}(t)$ into the CLIME algorithm (Cai et al., 2011).

More specifically, let $K(\cdot): \mathbb{R} \rightarrow \mathbb{R}$ be a symmetric nonnegative kernel function with support set $[-1,1]$. Moreover, for some absolute constant $C_{1}$, let $K(\cdot)$ satisfy that:

$$
\sup _{v} K(v) \leq C_{1}, \quad \int_{-1}^{1} K(v) d v=1, \quad \text { and } \quad \int_{0}^{1} v K(v) d v \leq C_{1} .
$$

Equation (2.2) is satisfied by a number of commonly used kernel functions. Examples include:

- Uniform kernel: $K(u)=I(|u| \leq 1) / 2$;

- Triangular kernel: $K(u)=(1-|u|) I(|u| \leq 1)$;

- Epanechnikov kernel: $K(u)=3\left(1-u^{2}\right) I(|u| \leq 1) / 4$; 
- Cosine kernel: $K(u)=\pi \cos (\pi u / 2) I(|u| \leq 1) / 4$.

For estimating any covariance matrix $\boldsymbol{\Sigma}\left(t_{0}\right)$ with the label $t_{0} \in[0,1]$, the smoothed sample covariance matrix estimator $\mathbf{S}\left(t_{0}\right)$ is calculated as follows:

$$
\mathbf{S}\left(t_{0}\right):=\sum_{i=1}^{n} \omega_{i}\left(t_{0}, h\right) \widehat{\mathbf{\Sigma}}_{i}
$$

where $\omega_{i}\left(t_{0}, h\right)$ is a weight function and $\widehat{\boldsymbol{\Sigma}}_{i}$ is the sample covariance matrix of $\boldsymbol{x}_{i 1}, \ldots, \boldsymbol{x}_{i m}$ :

$$
\omega_{i}\left(t_{0}, h\right):=\frac{c\left(t_{0}\right)}{n h} K\left(\frac{t_{i}-t_{0}}{h}\right), \quad \widehat{\boldsymbol{\Sigma}}_{i}:=\frac{1}{m} \sum_{l=1}^{m} \boldsymbol{x}_{i l} \boldsymbol{x}_{i l}^{T} \in \mathbb{R}^{d \times d} .
$$

Here $c\left(t_{0}\right)=2 I\left(t_{0} \in\{0,1\}\right)+I\left\{t_{0} \in(0,1)\right\}$ is a constant depending on whether $t_{0}$ is on the boundary or not, and $h$ is the bandwidth parameter. We will discuss how to select $h$ in the next section.

After obtaining the covariance matrix estimate, $\mathbf{S}\left(t_{0}\right)$, we consider estimating $\boldsymbol{\Omega}\left(t_{0}\right):=$ $\left\{\boldsymbol{\Sigma}\left(t_{0}\right)\right\}^{-1}$. When a suitable sparsity assumption on the inverse covariance matrix $\Omega\left(t_{0}\right)$ is available, we propose to estimate $\Omega\left(t_{0}\right)$ by plugging $\mathbf{S}\left(t_{0}\right)$ into the CLIME algorithm (Cai et al., 2011). In detail, the inverse covariance matrix estimator $\widehat{\Omega}\left(t_{0}\right)$ of $\boldsymbol{\Omega}\left(t_{0}\right)$ is calculated via solving the following optimization problem:

$$
\widehat{\Omega}\left(t_{0}\right)=\underset{\mathbf{M} \in \mathbb{R}^{d \times d}}{\operatorname{argmin}} \sum_{j k}\left|\mathbf{M}_{j k}\right|, \quad \text { subject to }\left\|\mathbf{S}\left(t_{0}\right) \mathbf{M}-\mathbf{I}_{d}\right\|_{\max } \leq \lambda,
$$

where $\mathbf{I}_{d} \in \mathbb{R}^{d \times d}$ is the identity matrix and $\lambda$ is the tuning parameter. Equation (2.5) can be further decomposed into $d$ minimization subproblems (Cai et al., 2011). For $j=1, \ldots, d$, the $j$-th column of $\widehat{\Omega}\left(t_{0}\right)$ can be solved as:

$$
\left\{\widehat{\boldsymbol{\Omega}}\left(t_{0}\right)\right\}_{* j}=\underset{\boldsymbol{v} \in \mathbb{R}^{d}}{\operatorname{argmin}}\|\boldsymbol{v}\|_{1}, \quad \text { subject to }\left\|\mathbf{S}\left(t_{0}\right) \boldsymbol{v}-\boldsymbol{e}_{j}\right\|_{\infty} \leq \lambda,
$$

where $\boldsymbol{e}_{j}$ is the $j$-th canonical vector. Equation (2.6) can be solved efficiently by applying a parametric simplex algorithm (Pang et al., 2013) and Equation (2.5) hence has the potential to be computed in parallel.

Once $\widehat{\Omega}\left(t_{0}\right)$ is obtained, we can apply an additional threshold step to estimate the Graph $\mathbf{G}\left(t_{0}\right)$. We define a graph estimator $\widehat{\mathbf{G}} \in\{0,1\}^{d \times d}$ to be:

$$
\left\{\widehat{\mathbf{G}}\left(t_{0}\right)\right\}_{j k}=\left\{\begin{array}{cc}
1 & \text { if }\left\{\widehat{\Omega}\left(t_{0}\right)\right\}_{j k}>\gamma \\
0 & \text { otherwise. }
\end{array}\right.
$$

Here $\gamma$ is another tuning parameter. Empirically, we find that the CLIME procedure has been working very efficiently in graph estimation without using this second thresholding (i.e., simply setting $\gamma=0$ ). 


\section{Theoretical Properties}

In this section the theoretical properties of the proposed estimators shown in Equations (2.5) and (2.7) are provided. Under a double asymptotic framework, the rates of convergence in parameter estimation under the matrix $\ell_{1}$ and $\ell_{\max }$ norms are given.

Before establishing the theoretical result, we first pose an additional assumption on the function $\Sigma(\cdot)$. In detail, let $\Sigma_{j k}(\cdot): t \rightarrow\{\Sigma(t)\}_{j k}$ be a real function. In the following, we assume that $\Sigma_{j k}(\cdot)$ is a smooth function with regard to any $j, k \in\{1, \ldots, d\}$. Here and in the sequel, the derivatives at support boundaries are defined as one-sided derivatives.

(A1) There exists one absolute constant, $C_{2}$, such that for all $t \in[0,1]$,

$$
\left|\frac{d}{d t} \Sigma_{j k}(t)\right| \leq C_{2}, \quad \text { for } j, k \in\{1, \ldots, d\} .
$$

Under Assumption (A1), we propose the following lemma, which shows that when the subjects observed are in $t=t_{1}, \ldots, t_{n}$ with $t_{i}=i / n$ for $i=1, \ldots, n$, the estimator $\mathbf{S}\left(t_{0}\right)$ approximates $\Sigma\left(t_{0}\right)$ at a fast rate for any $t_{0} \in[0,1]$. The convergence rate delivered here characterizes both the strength one can borrow across different subjects and the impact of temporal dependence structure on estimation accuracy.

Lemma 3.1. Suppose that the data points are generated from the model discussed in Section 2.1 and Assumption (A1) holds. Moreover, suppose that the observed subjects are in $t_{i}=$ $i / n$ for $i=1, \ldots, n$. Then, for any $t_{0} \in[0,1]$, if some $\eta>0$ we have

$$
\text { (A2) } \sup _{t \in[0,1]} \frac{d^{2}}{d t^{2}}\left\{K\left(\frac{t-t_{0}}{h}\right) \Sigma_{j k}(t)\right\}=O\left(h^{-\eta}\right), \quad \text { for } j, k \in\{1, \ldots, d\},
$$

and the bandwidth $h$ is set as

$$
h \asymp \max \left\{\left\{\frac{\xi \cdot \sup _{t \in[0,1]}\|\mathbf{\Sigma}(t)\|_{2}}{1-\sup _{t \in[0,1]}\|\mathbf{A}(t)\|_{2}} \sqrt{\frac{\log d}{m n}}\right\}^{1 / 2}, n^{-\frac{2}{2+\eta}}\right\} \text {, where } \xi:=\sup _{t \in[0,1]} \frac{\max _{j}[\boldsymbol{\Sigma}(t)]_{j j}}{\min _{j}[\boldsymbol{\Sigma}(t)]_{j j}}
$$

then the smoothed sample covariance matrix estimator $\mathbf{S}\left(t_{0}\right)$ in Equation (2.3) satisfies:

$$
\left\|\mathbf{S}\left(t_{0}\right)-\boldsymbol{\Sigma}\left(t_{0}\right)\right\|_{\max }=O_{P}\left[\left\{\frac{\xi \sup _{t \in[0,1]}\|\mathbf{\Sigma}(t)\|_{2}}{1-\sup _{t \in[0,1]}\|\mathbf{A}(t)\|_{2}} \sqrt{\frac{\log d}{m n}}\right\}^{1 / 2}+n^{-\frac{2}{2+\eta}}\right] .
$$

Assumption (A2) is a convolution between the smoothness of $K(t)$ and $\Sigma_{j k}(t)$, and is a weaker requirement than imposing smoothness individually. Assumption (A2) is satisfied 
by many commonly used kernel functions, including the aforementioned examples in Section 2.2. For example, with regard to the Epanechnikov kernel $K(u)=3\left(1-u^{2}\right) I(|u| \leq 1) / 4$, it's easy to check that

$$
\frac{d}{d t} K\left(\frac{t-t_{0}}{h}\right)=O\left(\frac{1}{h^{2}}\right) \quad \text { and } \quad \frac{d^{2}}{d t^{2}} K\left(\frac{t-t_{0}}{h}\right)=O\left(\frac{1}{h^{2}}\right) .
$$

Therefore, as long as $\Sigma_{j k}(t), \frac{d}{d t} \Sigma_{j k}(t)$, and $\frac{d^{2}}{d t^{2}} \Sigma_{j k}(t)$ are uniformly bounded, the Epanechnikov kernel satisfies Assumption (A2) with $\eta \geq 2$.

There are several observations drawn from Lemma 3.1. First, the rate of convergence in parameter estimation is upper bounded by $n^{-\frac{2}{2+\eta}}$, which is due to the bias in estimating $\Sigma\left(t_{0}\right)$ from only $n$ labels. This term is irrelevant to the sample size $m$ in each subject and cannot be improved without adding stronger (potentially unrealistic) assumptions. Secondly, the $m$ in $\{\log d /(m n)\}^{1 / 4}$ characterizes the strength one can borrow across different subjects. For example, when $\xi$, $\sup _{t}\|\boldsymbol{\Sigma}(t)\|_{2}$, and $\sup _{t}\|\mathbf{A}(t)\|_{2}$ all do not scale with $(n, m, d)$ and $m>C n^{\frac{6-\eta}{2+\eta}}$ for some generic constant $C$, the estimator achieves the $n^{-2 /(2+\eta)}$ rate of convergence. The first two points discussed above, together, quantify the settings where the proposed methods can beat the naive method which only uses the data points in each subject itself for parameter estimation. Finally, the term $1-\sup _{t \in[0,1]} \mathbf{A}(t)$ characterizes the impact of temporal dependence structure on estimation accuracy.

Consider now the case where $\mathbf{A}(t)=0$ and hence $\left\{\boldsymbol{x}_{i l}\right\}_{l=1}^{m}$ are independent observations without temporal dependences. In this case, following Zhou et al. (2010), the rate of convergence in parameter estimation for the proposed approach can be improved.

Lemma 3.2. Under the assumptions in Lemma 3.1, if it is further assumed that

(B1) $\left\{\boldsymbol{x}_{i l}\right\}_{l=1}^{m}$ are i.i.d. observations from $N_{d}\{\mathbf{0}, \boldsymbol{\Sigma}(t)\}$;

(B2) $\sup _{t \in[0,1]} \frac{d^{2}}{d t^{2}}\left[K^{2}\left(\frac{t-t_{0}}{h}\right)\left\{\Sigma_{j j}^{2}(t) \Sigma_{k k}^{2}(t)+\Sigma_{j k}^{2}(t)\right\}\right]=O\left(h^{-4}\right), \quad$ for all $j, k \in\{1, \ldots, d\}$;

(B3) There exists an absolute constant $C_{3}$ such that

$$
\max _{j k} \sup _{t \in[0,1]}\left|\Sigma_{j k}(t)\right| \leq C_{3}, \quad \max _{j k} \sup _{t \in[0,1]}\left|\frac{d}{d t} \Sigma_{j k}(t)\right| \leq C_{3}
$$

then, setting the bandwidth

$$
h \asymp \max \left\{\left(\frac{\log d}{m n}\right)^{1 / 3}, \frac{1}{n^{2 /(2+\eta)}}\right\},
$$


we have

$$
\left\|\mathbf{S}\left(t_{0}\right)-\mathbf{\Sigma}\left(t_{0}\right)\right\|_{\max }=O_{P}\left\{\left(\frac{\log d}{m n}\right)^{1 / 3}+n^{-\frac{2}{2+\eta}}\right\} .
$$

We note again that the aforementioned kernel functions satisfy Assumptions (B2) for similar reasons. In detail, taking Epanechnikov kernel as an example, we have

$$
\frac{d}{d t} K^{2}\left(\frac{t-t_{0}}{h}\right)=O\left(\frac{1}{h^{4}}\right), \quad \frac{d^{2}}{d t^{2}} K^{2}\left(\frac{t-t_{0}}{h}\right)=O\left(\frac{1}{h^{4}}\right) .
$$

So Assumption (B2) is satisfied as long as $\Sigma_{j k}(t), \frac{d}{d t} \Sigma_{j k}(t)$, and $\frac{d^{2}}{d t^{2}} \Sigma_{j k}(t)$ are uniformly bounded.

Lemma 3.2 shows that the rate of convergence can be improved to $\{\log d /(m n)\}^{1 / 3}$ when the data are independent. Of note, this rate matches the results in Zhou et al. (2010). However, the improved rate is valid only when a strong independent assumption holds, which is unrealistic in many applications, rs-fMRI data analysis for example.

After obtaining Lemmas 3.1 and 3.2, we proceed to the final result, which shows the theoretical performance of the estimator $\widehat{\Omega}\left(t_{0}\right)$ proposed in Equation (2.5). We show that under sparsity constraints, the proposed estimator is consistent, even when $d$ is nearly exponentially larger than $n$ and $m$.

Additional notations are first. Let $M_{d} \in \mathbb{R}$ be a quantity which may scale with $(n, m, d)$. We define the set of positive definite matrices in $\mathbb{R}^{d \times d}$, denoted by $\mathcal{M}\left(q, s, M_{d}\right)$, as

$$
\mathcal{M}\left(q, s, M_{d}\right):=\left\{\mathbf{M} \in \mathbb{R}^{d \times d}: \max _{1 \leq k \leq d} \sum_{j=1}^{d}\left|M_{j k}\right| \leq s,\|\mathbf{M}\|_{1} \leq M_{d}\right\} .
$$

For $q=0$, the class $\mathcal{M}\left(0, s, M_{d}\right)$ contains all the matrices with the number of nonzero entries in each column less than $s$ and bounded $\ell_{1}$ norm. We then let

$$
\begin{aligned}
& \kappa(n, m, d):=\left\{\frac{\xi \sup _{t \in[0,1]}\|\boldsymbol{\Sigma}(t)\|_{2}}{1-\sup _{t \in[0,1]}\|\mathbf{A}(t)\|_{2}} \sqrt{\frac{\log d}{m n}}\right\}^{1 / 2}+n^{-\frac{2}{2+\eta}} . \\
& \kappa^{*}(n, m, d):=\left(\frac{\log d}{m n}\right)^{1 / 3}+n^{-\frac{2}{2+\eta}} .
\end{aligned}
$$

Theorem 3.3 presents the parameter estimation and graph estimation consistency results for the estimator defined in Equations (2.5) and (2.7).

Theorem 3.3. Suppose that the conditions in Lemma 3.1 hold. Provided that $\boldsymbol{\Theta}\left(t_{0}\right):=$ $\left\{\boldsymbol{\Sigma}\left(t_{0}\right)\right\}^{-1} \in \mathcal{M}\left(q, s, M_{d}\right)$ with $0 \leq q<1$. Let $\widehat{\boldsymbol{\Theta}}\left(t_{0}\right)$ be defined in Equation (2.5). Then there exists a constant $C_{3}$ only depending on $q$, such that, whenever the tuning parameter

$$
\lambda=C_{3} M_{d} \cdot \kappa(n, m, d)
$$


is chosen, one has that

$$
\left\|\widehat{\boldsymbol{\Theta}}\left(t_{0}\right)-\boldsymbol{\Theta}\left(t_{0}\right)\right\|_{2}=O_{P}\left\{M_{d}^{2-2 q} \cdot s \cdot \kappa(n, m, d)^{(1-q) / 2}\right\} .
$$

Moreover, letting $\widehat{\mathbf{G}}\left(t_{0}\right)$ be the graph estimator defined in Equation (2.7) with the second step tuning parameter $\gamma=4 M_{d} \lambda$. If it is further assumed that $\boldsymbol{\Theta}\left(t_{0}\right) \in \mathcal{M}\left(0, s, M_{d}\right)$ and

$$
\min _{\left\{j, k:\left|\left\{\Theta\left(t_{0}\right)\right\}_{j k}\right| \neq 0\right\}}\left|\left\{\Theta\left(t_{0}\right)\right\}_{j k}\right| \geq 2 \gamma
$$

then

$$
\mathbb{P}\left\{\widehat{\mathbf{G}}\left(t_{0}\right)=\mathbf{G}\left(t_{0}\right)\right\}=1-o(1) .
$$

If the conditions in Lemma 3.2 hold, the above upper bound is true with $\kappa$ replaced by $\kappa^{*}$.

Theorem 3.3 shows that the proposed method is theoretically guaranteed to be consistent in both parameter estimation and model selection, even when the dimension $d$ is nearly exponentially larger than $n \cdot m$. Theorem 3.3 can be proved by following the proofs of Theorem 1 and Theorem 7 in Cai et al. (2011) and the proof is accordingly omitted.

\section{Experiments}

In this section, the empirical performance of the proposed method is investigated. This section is divided into two parts. In the first, the proposed kernel based method is compared to several existing methods on synthetic data, where the true generating models are known. The advantage of this new method is shown in both parameter estimation and model selection. In the second part, the proposed method is applied to a large scale resting state fMRI data (the ADHD-200 data) and some potentially scientifically interesting results are explored.

\subsection{Synthetic Data}

The performances of the proposed kernel-smoothing estimator (denoted as KSE) are compared to three existing methods: a naive estimator (donated as naive; details follow below), Danaher et al. (2013)'s group graphical lasso (denoted as GGL), and Guo et al. (2011)'s estimator. Throughout the simulation studies, it is assumed that the graphs are evolving from $t=0$ to $t=1$ continuously. The number of nodes in the graph is fixed to be 50 . Although there will be one graphical model corresponding to each $t \in[0,1]$, it is assumed that data are observed at points $t \in\{0,1 / 50,2 / 50, \ldots, 1\}$. For each $t \in\{0,1 / 50,2 / 50, \ldots, 1\}$, 
$m=100$ observations were generated from the corresponding graph under a stationary VAR(1) model discussed in Equation (2.1). To generate the transition matrix, $\mathbf{A}$, the precision matrix was obtained using the $\mathrm{R}$ package Huge (Zhao et al., 2012) with graph structure setting of "random". Then it is divided by twice its largest eigenvalue to obtain $\mathbf{A}$, so that $\|\mathbf{A}\|_{2}=0.5$. The same transition matrix is used under every label $t$. Our target is to estimate the graph at $t=0$, as the endpoints represent the most difficult point for estimation.

In the following, the three existing methods for comparison will be reviewed. The naive estimator is obtained by first calculating the sample covariance matrix at $t=0$ using only the $m=100$ observations under this label, and then plugged into the CLIME algorithm. Compared to KSE, Danaher et al. (2013)'s group graphical lasso and Guo et al. (2011)'s estimator do not assume that there exists a smooth change among the graphs. Instead, it is assumed that the data come from $n$ categories. That is, there are $n$ corresponding underlying graphs that potentially share common edges, and observations are available within each category. Moreover, they assume that the observations are independent both between and within different categories. With regard to implementation, they solve the following optimization problem:

$$
\max _{\boldsymbol{\Omega}^{(0)}, \ldots, \boldsymbol{\Omega}^{(n)} \succ 0} \sum_{i=0}^{n} m\left\{\log \operatorname{det} \boldsymbol{\Omega}^{(i)}-\operatorname{trace}\left(\widehat{\boldsymbol{\Sigma}}_{i} \boldsymbol{\Omega}^{(i)}\right)\right\}-P\left(\boldsymbol{\Omega}^{(0)}, \ldots, \boldsymbol{\Omega}^{(n)}\right),
$$

where $\widehat{\boldsymbol{\Sigma}}_{i}$ is the sample covariance matrix calculated based on the data under label $t_{i}$. The group graphical lasso uses penalty $P\left(\Omega^{(0)}, \ldots, \boldsymbol{\Omega}^{(n)}\right):=\lambda_{1} \sum_{i=0}^{n} \sum_{j \neq k}\left|\left\{\boldsymbol{\Omega}^{(i)}\right\}_{j k}\right|+$ $\lambda_{2} \sum_{j \neq k} \sqrt{\sum_{i=0}^{n}\left\{\boldsymbol{\Omega}^{(i)}\right\}_{j k}^{2}}$ and Guo et al. (2011)'s estimator uses penalty $P\left(\boldsymbol{\Omega}^{(0)}, \ldots, \boldsymbol{\Omega}^{(n)}\right):=$ $\lambda \sum_{j \neq k} \sqrt{\sum_{i=0}^{n}\left|\left\{\boldsymbol{\Omega}^{(i)}\right\}_{j k}\right|}$. Here the regularity coefficients $\lambda_{1}, \lambda_{2}$, and $\lambda$ control the sparsity level. Danaher et al. (2013) also proposed the fused graphical lasso that seperately controls sparsity of and the similarity between the graphs. However, this method is not scalable when the number of categories is large and therefore do not include it into comparison.

After obtaining the estimated graph, $\widehat{\mathbf{G}}\left(t_{0}\right)$, of the true traph $\mathbf{G}\left(t_{0}\right)$, the model selection performance is further investigated by comparing the ROC curves of the four competing methods. Let $\widehat{\mathrm{E}}\left(t_{0}\right)$ be the set of estimated edges corresponding to $\widehat{\mathbf{G}}\left(t_{0}\right)$, and $\mathrm{E}\left(t_{0}\right)$, the set of true edges corresponding to $\mathbf{G}\left(t_{0}\right)$. The true positive rate (TPR) and false positive rate $(\mathrm{FPR})$ are defined as

$$
\operatorname{TPR}=\frac{\left|\widehat{\mathrm{E}}\left(t_{0}\right) \bigcap \mathrm{E}\left(t_{0}\right)\right|}{\left|\mathrm{E}\left(t_{0}\right)\right|}, \quad \mathrm{FPR}=\frac{\left|\widehat{\mathrm{E}}\left(t_{0}\right) \backslash \mathrm{E}\left(t_{0}\right)\right|}{d(d-1) / 2-\left|\mathrm{E}\left(t_{0}\right)\right|},
$$

where for any set $S,|S|$ denotes the cardinality of the set $S$. To obtain a series of TPRs and FPRs, for KSE, naive estimator, and Guo et al. (2011)'s methods, the values of $\lambda$ are 
varied. For GGL, first $\lambda_{2}$ is fixed and subsequently $\lambda_{1}$ is tuned, and then the $\lambda_{2}$ with the best overall performance is selected. More specifically, a series of $\lambda_{2}$ are picked, and for each fixed $\lambda_{2}, \lambda_{1}$ is accordingly varied to produce an ROC curve. Of note, in the investigation, the ROC curves indexed by $\lambda_{2}$ are generally parallel, thus motivating this strategy. Finally, the $\lambda_{2}$ corresponding to the topleft most curve is selected.

\subsubsection{Setting 1: Simultaneously Evolving Edges}

In this section we investigate the performances of the four competing methods under one particular graphical model. We illustrate the generating model under this setting as follows: In each simulation, $n_{\mathrm{fix}}=200$ edges are randomly selected from $d(d-1) / 2$ potential edges and they do not change with regard to the label $t$. The strengths of these edges, i.e. the corresponding entries in the inverse covariance matrix, are generated from a uniform distribution taking ranges in $[-0.3,-0.1]$ (denoted by Unif $[-0.3,-0.1]$ ) and do not change with $t$. We then randomly select $n_{\text {decay }}$ and $n_{\text {grow }}$ edges that will disappear and emerge over the evolution simultaneously. For each of the $n_{\text {decay }}$ edges, the strength is generated from Unif[-0.3,-0.1] at $t=0$ and will diminish to 0 linearly with regard to $t$. For each of the $n_{\text {grow }}$ edges, the strength is set to be 0 at $t=0$, and will linearly grow to a value generated from Unif[-0.3,-0.1]. The edges evolve simultaneously. For $j \neq k$, when we subtract a value $a$ from $\boldsymbol{\Omega}_{j k}$ and $\boldsymbol{\Omega}_{k j}$, we increase $\boldsymbol{\Omega}_{j j}$ and $\boldsymbol{\Omega}_{k k}$ by $a$, and then further add 0.25 to the diagonal of the matrix to keep it positive definite.

The ROC curves under this setting with different values of $n_{\text {grow }}$ and $n_{\text {decay }}$ are shown in Figures 1(a) and 1(b). It can be observed that, under both cases, KSE outperforms the other three competing methods. Moreover, when we increase the values of $n_{\text {grow }}$ and $n_{\text {decay }}$ from 20 to 100, the ROC curve of KSE hardly changes, since the degree of smoothness in graphical model evolving hardly change. In contrast, the ROC curves of GGL and Guo et al. (2011) drop, since the degree of similarity among the graphs is reduced. Finally, naive's performance is very bad, which is expected because it cannot borrow strength in estimation from other subjects.

Next, we exploit the same data, but permute the labels $t=1 / 50,2 / 50, \ldots, 1$ so that the evolving pattern is much more opaque. Figures $1(\mathrm{c})$ and 1(d) illustrate the model selection result. We observe that under this setting, the ROC curves of the proposed method drop a little bit, but is still higher than the competing approaches. This is probably because the proposed method can still benefit from the evolving graph structure (although more turbulent this time). The improvement over the naive method demonstrates exactly the strength we have borrowed across different labels. Note that the ROC curves of GGL, naive, 


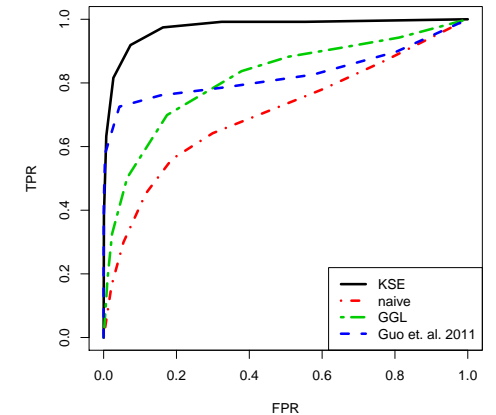

(a) Setting $1 ; n_{\text {grow }}=n_{\text {decay }}=20$

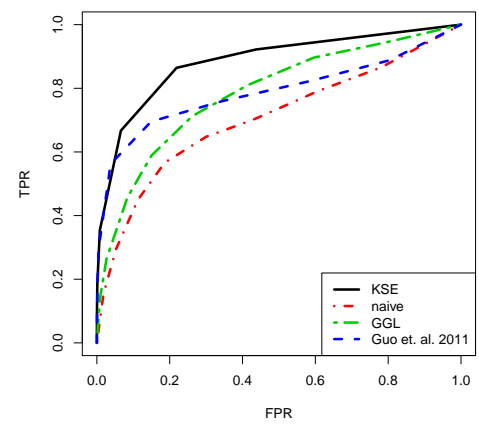

(d) Setting 1 with permutation; $n_{\text {grow }}=n_{\text {decay }}=100$

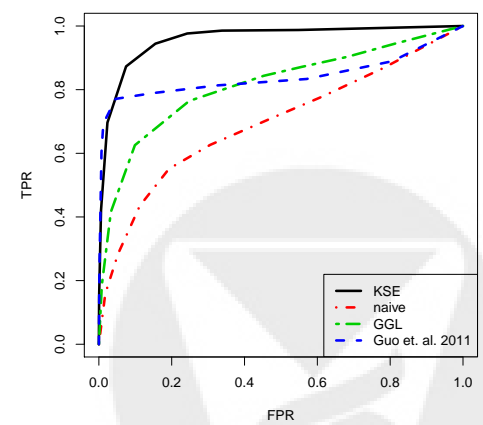

(g) Setting 2 with permutation; (h) Setting 2 with permutation; $n_{\text {grow }}=40$

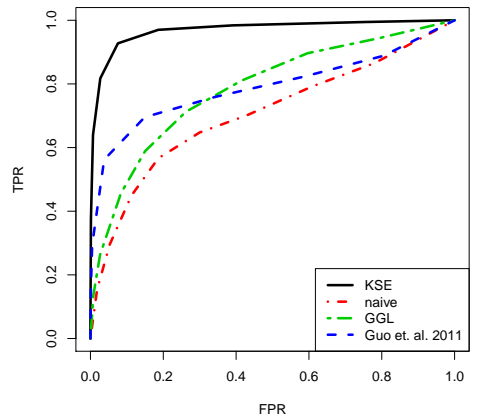

(b) Setting $1 ; n_{\text {grow }}=n_{\text {decay }}=100$

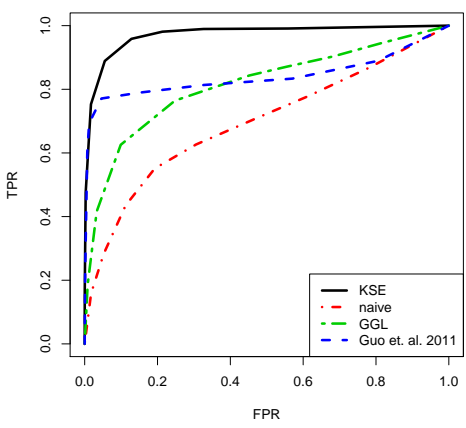

(e) Setting $2 ; n_{\text {grow }}=40$

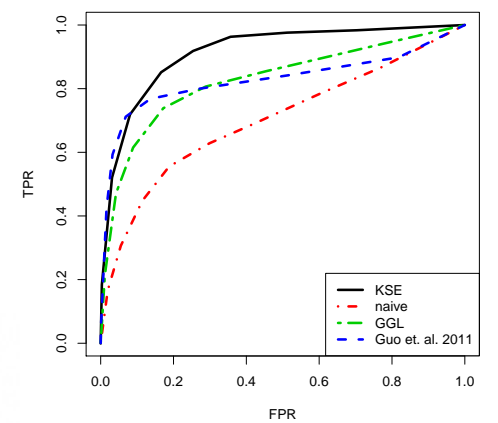

$n_{\text {grow }}=200$ (c) Setting 1 with permutation;

$$
n_{\text {grow }}=n_{\text {decay }}=20
$$
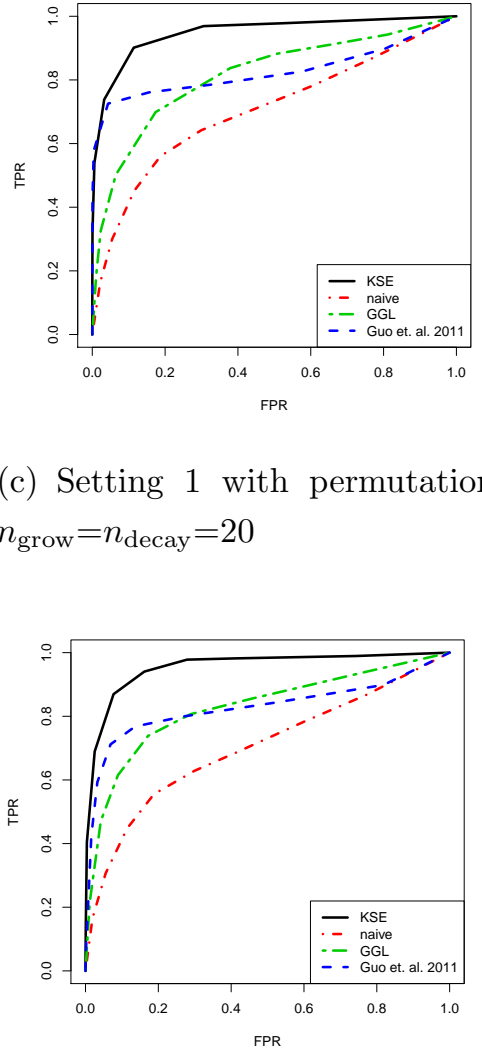

(f) Setting $2 ; n_{\text {grow }}=200$

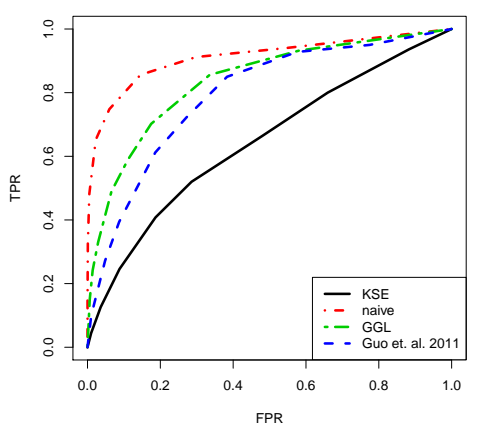

(i) Setting $3 ; n_{\text {grow }}=200$

Figure 1: ROC curves of four competing methods under three settings: simultaneous (a-d), sequential (e-h), and random (i). In each setting we set the dimension $d=50$, the number of labels $n=51$, the number of observations $m=100$, and the result is obtained by 1,000 simulations. 
and Guo et al. (2011) shown in Figures1(c) and 1(d) do not change with regard to that in Figures 1(a) and 1(b) separately, because they do not assume any ordering between the graphs.

\subsubsection{Setting 2: Sequentially Growing Edges}

Setting 2 is similar to Setting 1. The two differences are: (i) Here $n_{\text {decay }}$ is set to be zero;

(ii) The $n_{\text {grow }}$ edges emerges sequentially instead of simultaneously. These $n_{\text {grow }}$ edges are randomly selected, but there is no overlap with the existing 200 pre-fixed edges. The entries of the inverse covariance matrix for the $n_{\text {grow }}$ edges each grows to a value generated from Unif $[-0.3,-0.1]$, linearly in a sequence of length $1 / n_{\text {grow }}$ in the range $[0,1]$, and one after the other. We note that there is possibility that $n<n_{\text {grow }}$. It is because here $n$ represents only the labels that we can observes. Under this setting, Figures 1(e) and 1(f) plot the ROC curves of four competing methods. We also applied the four methods to the setting where the same permutation as in Setting 1 is exploited. We show the results in Figures $1(\mathrm{~g})$ and $1(\mathrm{~h})$. Here we find the same observations as what we found in Setting 1 take place.

\subsubsection{Setting 3: Random Edges}

In this setting, in contrast to the above two settings, we violate the smoothness assumption of KSE to the extreme. What we want to show in this setting is the limitedness of the proposed method. More specifically, in this setting, under every label $t, n_{\text {ed }}$ edges are random selected with strengths from Unif $[-0.3,-0.1]$. In this case, the graphs do not evolve smoothly over the label $t$, and the data under the labels $t \neq 0$ only contribute noises. We then apply the four competing methods to this setting and Figure 1(i) illustrates the result. Under this setting, we observe that naive beats all the other three methods. It is expected because naive is the only method that do not take noises into account for estimation. Here KSE performs worse than GGL and Guo et al. (2011), because there does not exist a natural ordering among the graphs.

Under the above three data generating settings, we further quantitatively compare the performance in parameter estimation of the inverse covariance matrix $\Omega\left(t_{0}\right)$ for the four competing methods. Here the distances between the estimated and the true concentration matrices with regard to the matrix $\ell_{1}, \ell_{2}$, and Frobenius norms are shown in Table 1 . It can be observed that KSE achieves the lowest estimation error in all settings except for 
the Setting 3. This coincides with the above model selection results. We do not show the label permutation cases under Setting 1 and Setting 2, but the same results as drawn in investigating the model selection performance also hold here.

Table 1: Comparison of inverse covariance matrix estimation errors in there data generating models. The parameter estimation error with regard to the matrix $\ell_{1}, \ell_{2}$, and Frobenius norms (denoted as $\ell_{F}$ here) is provided with standard deviations provided in the brackets. The results are obtained by 1,000 simulations.

\begin{tabular}{|c|c|c|c|c|c|c|c|}
\hline \multirow{4}{*}{ Setting 1} & \multirow[b]{2}{*}{$n_{\text {grow }}=n_{\text {decay }}$} & \multicolumn{3}{|c|}{ KSE } & \multicolumn{3}{|c|}{ naive } \\
\hline & & $\ell_{1}$ & $\ell_{2}$ & $\ell_{F}$ & $\ell_{1}$ & $\ell_{2}$ & $\ell_{F}$ \\
\hline & 20 & $3.25(0.232)$ & $1.53(0.104)$ & $4.42(0.220)$ & $5.02(0.287)$ & $2.68(0.132)$ & $8.30(0.412)$ \\
\hline & 100 & $2.72(0.165)$ & $1.30(0.088)$ & $3.78(0.204)$ & $4.85(0.467)$ & $2.55(0.117)$ & $8.13(0.453)$ \\
\hline \multirow{3}{*}{ Setting 2} & $n_{\text {grow }}$ & & & & & & \\
\hline & 40 & $3.39(0.553)$ & $1.56(0.213)$ & $4.47(0.302)$ & $5.26(0.740)$ & $2.73(0.313)$ & $8.24(0.386)$ \\
\hline & 200 & $3.40(0.507)$ & $1.57(0.147)$ & $4.33(0.284)$ & $5.19(0.740)$ & $2.71(0.280)$ & $8.34(0.352)$ \\
\hline \multirow{4}{*}{ Setting 3} & $n_{\mathrm{ed}}$ & & & & & & \\
\hline & 50 & $2.21(0.194)$ & $1.37(0.120)$ & $3.20(0.104)$ & $1.60(0.249)$ & $0.84(0.113)$ & $3.09(0.185)$ \\
\hline & & \multicolumn{3}{|c|}{ GGL } & \multicolumn{3}{|c|}{ Guo et. al. 2011} \\
\hline & $n_{\text {grow }}=n_{\text {decay }}$ & $\ell_{1}$ & $\ell_{2}$ & $\ell_{F}$ & $\ell_{1}$ & $\ell_{2}$ & $\ell_{F}$ \\
\hline \multirow[t]{2}{*}{ Setting 1} & 20 & $3.28(0.298)$ & $1.45(0.112)$ & $4.13(0.190)$ & $3.22(0.418)$ & $1.42(0.259)$ & $4.04(0.280)$ \\
\hline & 100 & $3.27(0.324)$ & $1.42(0.100)$ & $4.18(0.222)$ & $3.38(0.474)$ & $1.41(0.169)$ & $4.31(0.335)$ \\
\hline & $n_{\text {grow }}$ & & & & & & \\
\hline \multirow[t]{2}{*}{ Setting 2} & 40 & $3.47(0.580)$ & $1.47(0.163)$ & $4.22(0.153)$ & $3.06(0.417)$ & $1.40(0.274)$ & $4.00(0.205)$ \\
\hline & 200 & $3.22(0.618)$ & $1.44(0.198)$ & $4.08(0.199)$ & $3.71(0.493)$ & $1.73(0.264)$ & $4.46(0.361)$ \\
\hline \multirow{2}{*}{ Setting 3} & $n_{\mathrm{ed}}$ & & & & & & \\
\hline & 50 & $1.52(0.224)$ & $0.85(0.105)$ & $2.04(0.104)$ & $1.48(0.263)$ & $0.67(0.116)$ & $1.81(0.150)$ \\
\hline
\end{tabular}

\subsection{The Impact of a Small Label Size $n$}

As is shown in Lemma 3.1 and Theorem 3.3, the rates of convergence in parameter estimation and model selection crucially depend on the term $n^{-\frac{2}{2+\eta}}$. This is due to the bias 
in estimating $\boldsymbol{\Sigma}\left(t_{0}\right)$ from $n$ labels. This bias takes place as long as we include data under other labels into estimation, and cannot be removed by simply increasing the number of observations $m$ under each label $t$. More specifically, in the appendix, Lemma A.1 will show quantitatively that the rate of convergence for bias between the estimated and the true covariance matrix is only controlled by $n$, and cannot be eliminated by only increasing the number of observations under each label $m$ while holding $n$ fixed.

This section is devoted to illustrate this phenomenon empirically. We exploit Setting 2 in the last section with the number of labels $n$ to be a very small number. Here we set $n=3$. Moreover, we choose $n_{\text {fix }}=100, n_{\text {grow }}=500$, and change the number of observations $m$ under each label. Figure 2 compares the ROC curves of KSE and naive corresponding to the settings when $m=100$ or 500 . There are two important observations we would like to emphasize: (i) We find that, when $m=100$, KSE and naive's performances are comparable. However, when $m=500$, naive performs much better than KSE. (ii) The change of the ROC curves for KSE between $m=100$ and $m=500$ is not that dramatic compared to the ROC curves for naive. Ever since everything else in the model is fixed, these two observations indicate that the bias term in KSE indeed has effect in model selection.

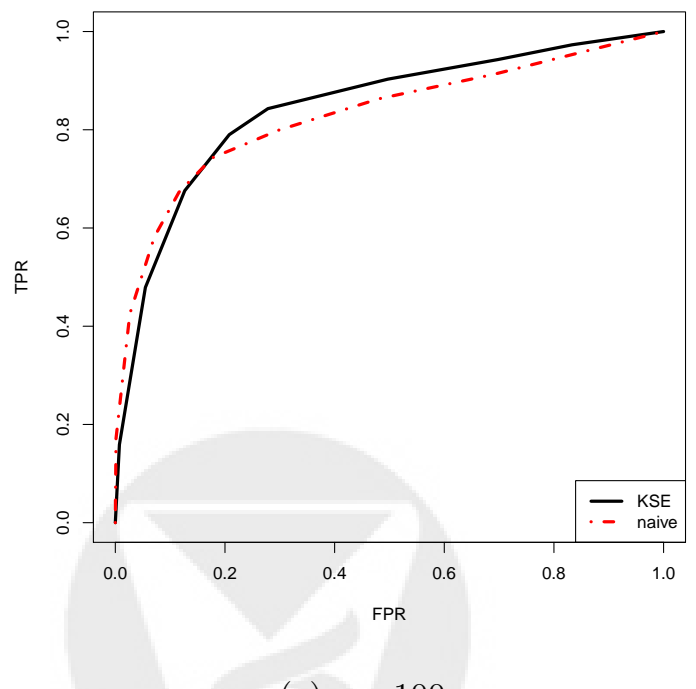

(a) $m=100$

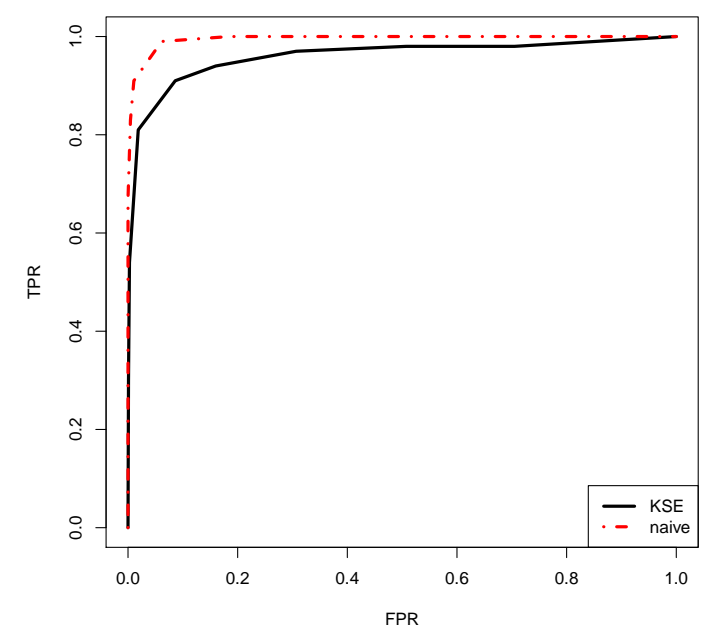

(b) $m=500$

Figure 2: ROC curves of KSE and naive under Setting 1: sequentially evolving edges. We set dimension $d=50$; number of labels $n=3$; number of pre-fixed edges $n_{\text {fix }=100}$; number of growing edges $n_{\text {grow }}=500$. 


\subsection{ADHD-200 Data}

As an example of real data application, we apply the proposed method to the ADHD-200 data (Biswal et al., 2010). The ADHD-200 data consist of resting state functional magnetic resonance imaging (rs-fMRI) images of 973 subjects. Of them, 491 are healthy and 197 have been diagnosed with ADHD type 1,2, or 3. The remaining had their diagnosis withheld for the purpose of a prediction competition. The number of images for each subject ranged from 76 to 276. 264 seed regions of interest were used to define nodes for graphical model analysis (Power et al., 2011). A limited set of covariates including gender, age, handedness, IQ, are available.

In this section focus lies on studying the brain development over age for control subjects to investigate network development. Here the age measurements were normalized to be in $[0,1]$, and the brain ROI measurements were centered to have sample means zero and scaled to sample standard deviations 1 . The bandwidth parameter was set at $h=0.5$ while the regularization parameter, $\lambda$, was fixed at 3,400 . The tuning parameter was set so high for better visualization and highlighting the dominating edges. Consider estimating the brain networks at the ages 7.09, 11.75, and 21.83, which are the minimal, median, and maximal ages in the data. Figure 3 shows coronal, sagittal, and transverse snapshots of the estimated brain connectivity networks.

There are two main patterns worth noting in this experiment: (i) 494, 878, and 1,032 edges are estimated at the three ages, and thus the number of edges grew dramatically with age. This is consistent with the hypothesis of increasing network complexity with development and greater network heterogeneity at younger ages. (ii) It is observed that the degree of complexity of the brain network at the occipital lobe is high compared to other regions by age seven. This is consistent with early maturation of visual and vision processing networks relative to others. We found that this conjecture is supported by several recent scientific results (Shaw et al., 2008; Blakemore, 2012). For example, Shaw et al. (2008) showed that occipital lobe is fully developed before other brain regions. Moreover, when considering structural development, the occipital lobe reaches its peak thickness by the age of nine. In comparison, portions of the parietal lob reaches their peak thickness as late as thirteen. We also noticed that several confounding factors, such as scanner noise, subject motion, and coregistration, can have potential effects on inference (Braun et al., 2012; Van Dijk et al., 2012). In this manuscript we rely on the standard data preprocessing techniques as described in Eloyan et al. (2012) for removing such confounders, but will investigate the influence of these confounders on our inference in more details in the future. 


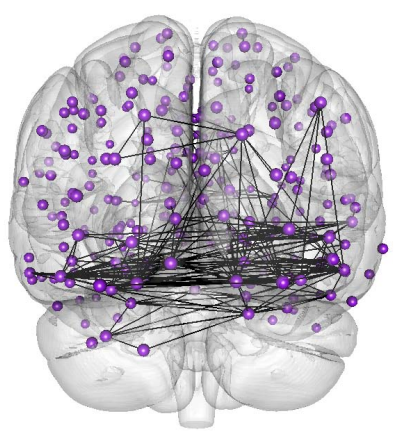

(a) coronal

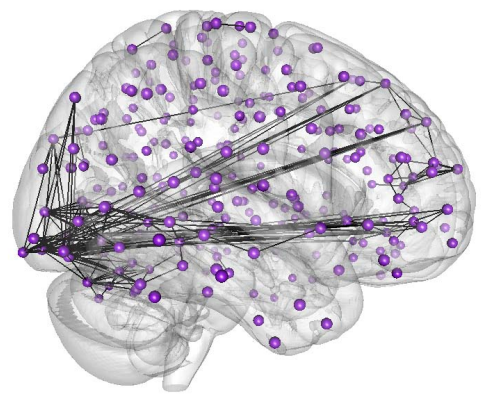

(b) sagittal

Age 7.09; 494 edges

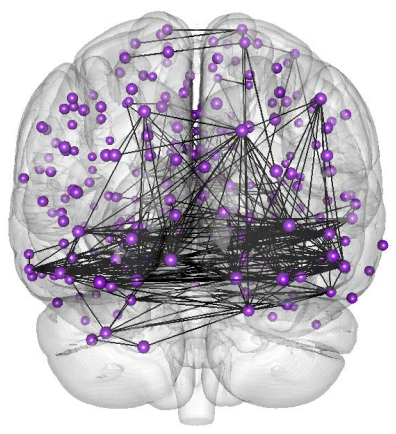

(d) coronal

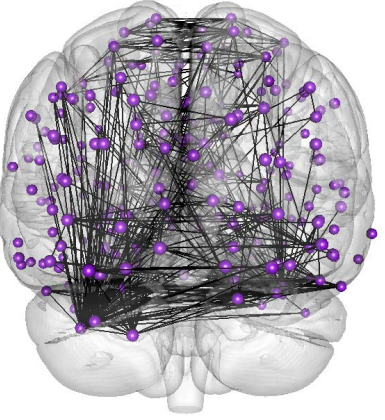

(g) coronal

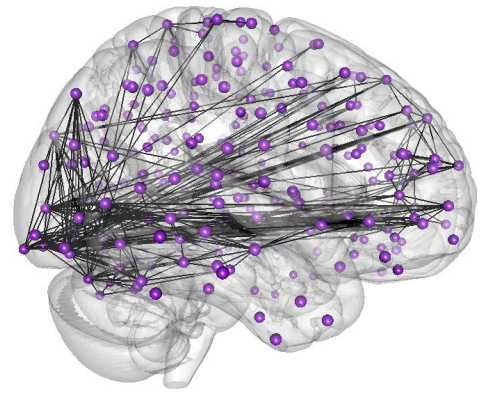

(e) sagittal

Age 11.75; 878 edges

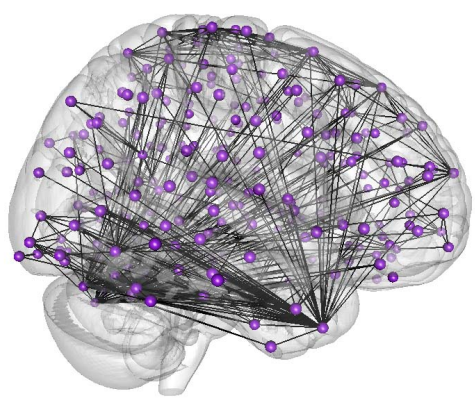

(h) sagittal

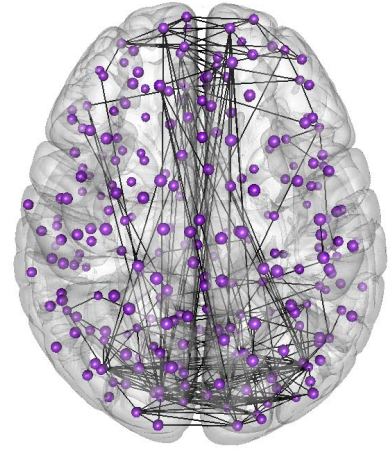

(c) transverse

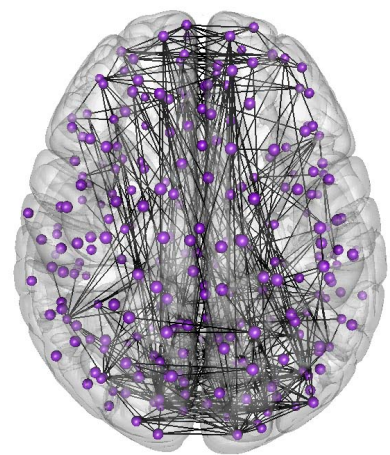

(f) transverse

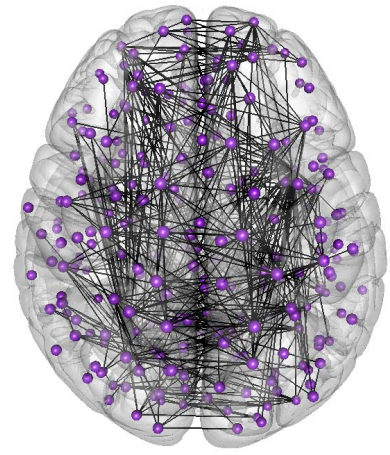

(i) transverse

Age 21.83; 1032 edges

Figure 3: Estimated brain connectivity network at the ages 7.09, 11.75, 21.83 among healthy subjects. of Blostallstics 
In the rest of this section, the impact of bandwidths on estimation is considered. In practice, the bandwidth can be regarded as the degree of tradeoff between the label-specific networks and the population level networks. Under such a logic, a higher value of bandwidth will result in incorporating more information from the data points in other labels, and lead to an estimate closer to a population-level graph. This population-level graph will highlight the similarity between different graphs, while tending to ignore the label-specific differences. To illustrate this phenomenon empirically, consider estimating the brain network at the age 21.83. We increase the bandwidth $h$, while setting all the other parameters fixed (e.g., $\lambda$ is fixed to be 3,400$)$. It can be observed that, as $h$ is increased from 0.5 to 3 , the weights in Equation (2.4) tends to be homogeneous across ages. Thus the graph ranges from agespecific level to the population level. Figure 4 plots the different brain connectivity graphs estimated using different bandwidths.

There are three main discoveries: (i) The number of edges decreases to a population level of 674 as $h$ increase to 3 . This is intuitive, because the population level brain network will summarize the information across different levels and thus should be more concrete. (ii) When $h=3$, the estimated brain network is close to the network estimated in the age 7.09 shown in Figure 3 with most edges taking place at the occipital lobe region. This is expected because the occipital lobe region is the only part that has been well developed across the entire range of ages. (iii) Another observation in Figure 3 is that dense connections in the temporal lobe only occur in the graph at age 21.83 among the ages shown. This is also supported by the scientific finding that grey matter in the temporal lobe doesn't reach maximum volume untill age 16 (Bartzokis et al., 2001; Giedd et al., 1999).

\section{Discussion}

In this paper we introduced a new kernel based estimator for jointly estimating multiple graphs under the conditions that the graphs smoothly change according to a label. Methodologically, motivated by resting state functional brain connectivity analysis, we proposed a new model, taking both heterogeneity structure and non-independent issues into consideration, and propose a new kernel based method under this model. Theoretically, we provided the model selection and parameter estimation consistency result for the proposed method under both the independent and non-independent assumptions. Empirically, we applied the proposed method to synthetic and brain image data. We found that the proposed method is effective for both parameter estimation and model selection compared to several existing methods under various settings. 


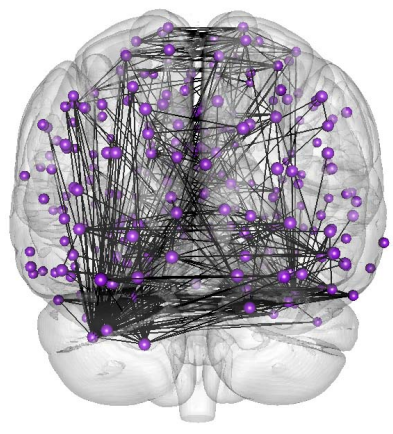

(a) coronal

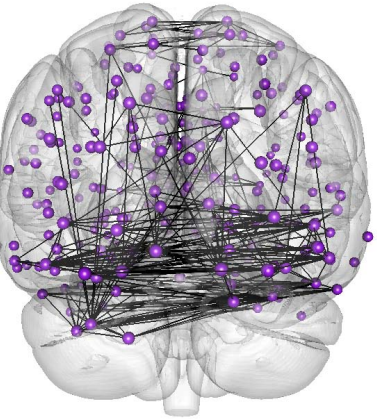

(d) coronal

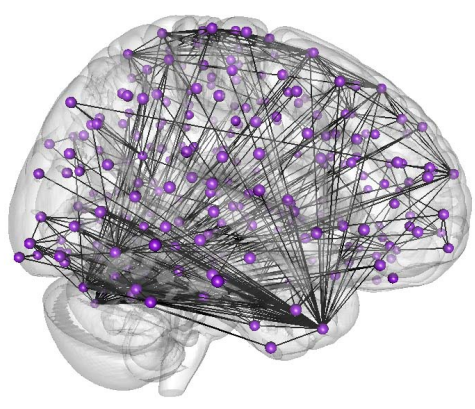

(b) sagittal

$$
h=0.5 ; 1032 \text { edges }
$$

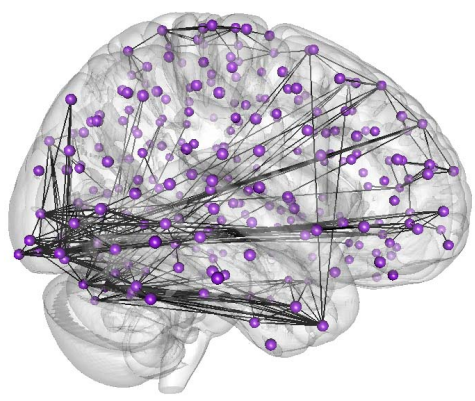

(e) sagittal

$$
h=1 ; 718 \text { edges }
$$

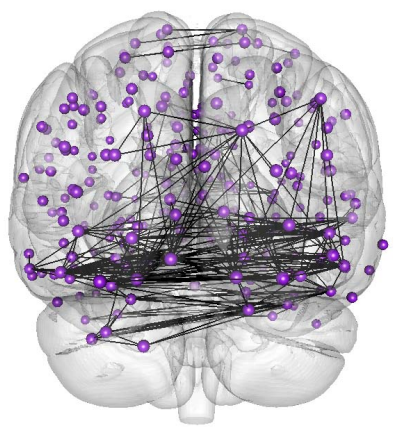

(g) coronal

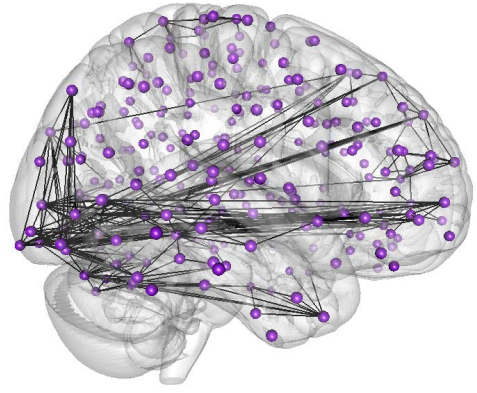

(h) sagittal

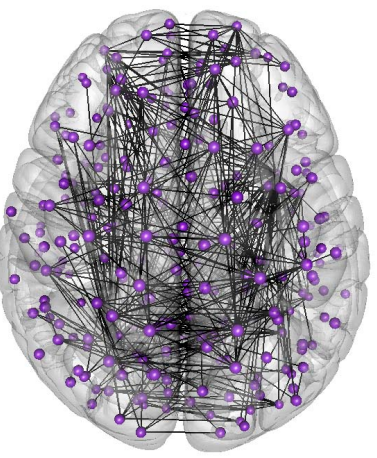

(c) transverse

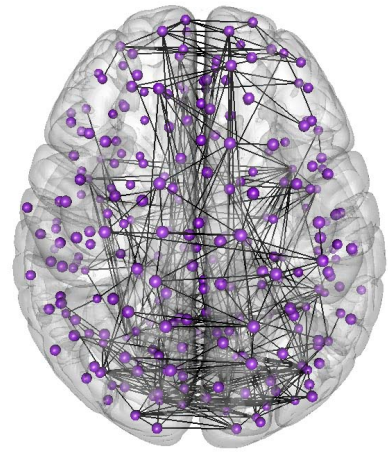

(f) transverse

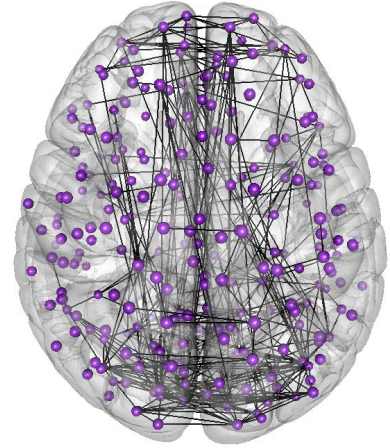

(i) transverse

$$
h=3 ; 674 \text { edges }
$$

Figure 4: Estimated brain connectivity network at the age 21.83 among healthy subjects. The kernel bandwidth $h$ takes the value $0.5,1,3$, resulting to different brain connectivity networks from closer to the age-specific level, to closer to the population level. 


\section{A Proof of Lemma 3.1}

The proof of Lemma 3.1 can be decomposed into two parts. In the first part, we prove that the bias term $\mathbb{E} \mathbf{S}\left(t_{0}\right)-\boldsymbol{\Sigma}\left(t_{0}\right)$ can be controlled by the number of subjects $n$ and bandwidth $h$. The result is provided in the following lemma.

Lemma A.1. Supposing that the conditions in Lemma 3.1 hold, we have

$$
\max _{j, k}\left|\mathbb{E}\left\{\mathbf{S}\left(t_{0}\right)\right\}_{j k}-\Sigma_{j k}\left(t_{0}\right)\right|=O\left(h+\frac{1}{n^{2} h^{1+\eta}}\right) .
$$

Proof. By the definition of $\mathbf{S}\left(t_{0}\right)$ in Equation (2.3), we have

$$
\mathbf{S}\left(t_{0}\right)=\sum_{i=1}^{n} \omega_{i}\left(t_{0}, h\right) \frac{1}{m} \sum_{k=1}^{m} \boldsymbol{x}_{i k} \boldsymbol{x}_{i k}^{T}
$$

Accordingly, we have

$$
\begin{aligned}
\mathbb{E}\left[\mathbf{S}\left(t_{0}\right)\right]_{j k} & =\sum_{i=1}^{n} \omega_{i}\left(t_{0}, h\right) \frac{1}{m} \sum_{k=1}^{m} \mathbb{E} \boldsymbol{x}_{i k} \boldsymbol{x}_{i k}^{T} \\
& =\sum_{i=1}^{n} \omega_{i}\left(t_{0}, h\right) \Sigma_{j k}\left(t_{i}\right) \\
& =\frac{c\left(t_{0}\right)}{n h} \sum_{i=1}^{n} K\left(\frac{t_{i}-t_{0}}{h}\right) \Sigma_{j k}\left(t_{i}\right) .
\end{aligned}
$$

By Theorem 1.1 in Tasaki (2009) and Assumption (A2), we have

$$
\begin{aligned}
& \frac{c\left(t_{0}\right)}{n h} \sum_{i=1}^{n} K\left(\frac{t_{i}-t_{0}}{h}\right) \Sigma_{j k}\left(t_{i}\right) \\
= & \frac{c\left(t_{0}\right)}{h} \int_{0}^{1} K\left(\frac{t-t_{0}}{h}\right) \Sigma_{j k}(t) d t+O\left[\frac{c\left(t_{0}\right)}{n^{2} h} \sup _{t \in[0,1]} \frac{d^{2}}{d t^{2}}\left\{K\left(\frac{t-t_{0}}{h}\right) \Sigma_{j k}(t)\right\}\right] \\
= & c\left(t_{0}\right) \int_{-\frac{t_{0}}{h}}^{\frac{1-t_{0}}{h}} K(u) \Sigma_{j k}\left(t_{0}+h u\right) d u+O\left(\frac{1}{n^{2} h^{1+\eta}}\right) \\
= & c\left(t_{0}\right) \int_{a\left(t_{0}\right)}^{b\left(t_{0}\right)} K(u)\left\{\Sigma_{j k}\left(t_{0}\right)+h u \Sigma_{j k}^{\prime}(\zeta)\right\} d u+O\left(\frac{1}{n^{2} h^{1+\eta}}\right),
\end{aligned}
$$

where $a\left(t_{0}\right):=-I\left(t_{0} \in(0,1]\right), b\left(t_{0}\right):=I\left(t_{0} \in[0,1)\right), \Sigma_{j k}^{\prime}(t):=\frac{d}{d t} \Sigma_{j k}(t)$, and $\zeta$ lies between $t_{0}$ and $t_{0}+h u$. The last equality is because $h \rightarrow 0$ and $K(u)$ has support $[-1,1]$.

By Equation (2.2), we have

$$
c\left(t_{0}\right) \int_{a\left(t_{0}\right)}^{b\left(t_{0}\right)} K(u) \Sigma_{j k}\left(t_{0}\right) d u=\Sigma_{j k}\left(t_{0}\right) .
$$


By Equation (2.2) and Assumption (A1), we have

$$
\begin{aligned}
\left|c\left(t_{0}\right) \int_{a\left(t_{0}\right)}^{b\left(t_{0}\right)} K(u) h u \Sigma_{j k}^{\prime}(\zeta) d u\right| & \leq C_{2} h\left|c\left(t_{0}\right) \int_{a\left(t_{0}\right)}^{b\left(t_{0}\right)}\right| u|K(u) d u| \\
& =2 C_{2} h\left|\int_{0}^{1} u K(u) d u\right|=O(h) .
\end{aligned}
$$

Combining (A.1), (A.2), (A.3), and (A.4), we have

$$
\left|\mathbb{E}\left\{\mathbf{S}\left(t_{0}\right)\right\}_{j k}-\Sigma_{j k}\left(t_{0}\right)\right|=O\left(h+\frac{1}{n^{2} h^{1+\eta}}\right) .
$$

This completes the proof.

We then proceed to the second lemma, which provides an upper bound of the distance between the estimator $\mathbf{S}\left(t_{0}\right)$ and its expectation $\mathbb{E} \mathbf{S}\left(t_{0}\right)$.

Lemma A.2. Supposing that the conditions in Lemma 3.1 hold, we have

$$
\max _{j, k}\left|\left\{\mathbf{S}\left(t_{0}\right)\right\}_{j k}-\mathbb{E}\left\{\mathbf{S}\left(t_{0}\right)\right\}_{j k}\right|=O_{P}\left[\frac{\xi \cdot \sup _{t \in[0,1]}\|\mathbf{\Sigma}(t)\|_{2}}{h\left\{1-\sup _{t \in[0,1]}\|\mathbf{A}(t)\|_{2}\right\}} \sqrt{\frac{\log d}{m n}}\right] .
$$

Proof. For $i=1, \ldots, n$ and $l=1, \ldots, m$, let $\boldsymbol{y}_{i l}:=\left(y_{i l 1}, \ldots, y_{i l d}\right)^{T}$ be a $d$-dimensional random vector with $y_{i l j}=x_{i l j} / \sqrt{\Sigma_{j j}\left(t_{i}\right)}$ and $\rho_{j k}\left(t_{i}\right):=\Sigma_{j k}\left(t_{i}\right) / \sqrt{\Sigma_{j j}\left(t_{i}\right) \Sigma_{k k}\left(t_{i}\right)}$. We then have

$$
\begin{aligned}
& \mathbb{P}\left[\left|\left\{\mathbf{S}\left(t_{0}\right)\right\}_{j k}-\mathbb{E}\left\{\mathbf{S}\left(t_{0}\right)\right\}_{j k}\right|>\epsilon\right] \\
= & \mathbb{P}\left[\left|\sum_{i=1}^{n} \omega_{i}\left(t_{0}, h\right)\left\{\frac{1}{m} \sum_{l=1}^{m} x_{i l j} x_{i l k}-\Sigma_{j k}\left(t_{i}\right)\right\}\right|>\epsilon\right] \\
= & \mathbb{P}\left\{\mid \sum_{i=1}^{n} \omega_{i}\left(t_{0}, h\right) \sqrt{\Sigma_{j j}\left(t_{i}\right) \Sigma_{k k}\left(t_{i}\right)}\left(\left[\frac{1}{m} \sum_{l=1}^{m}\left(y_{i l j}+y_{i l k}\right)^{2}-2\left\{1+\rho_{j k}\left(t_{i}\right)\right\}\right]\right.\right. \\
& \left.\left.-\left[\frac{1}{m} \sum_{l=1}^{m}\left(y_{i l j}-y_{i l k}\right)^{2}-2\left\{1-\rho_{j k}\left(t_{i}\right)\right\}\right]\right) \mid>4 \epsilon\right\} \\
\leq & \mathbb{P}\left\{\left|\sum_{i=1}^{n} \omega_{i}^{*}\left(t_{0}, h\right)\left(\left[\frac{1}{m} \sum_{l=1}^{m}\left(y_{i l j}+y_{i l k}\right)^{2}-2\left\{1+\rho_{j k}\left(t_{i}\right)\right\}\right]\right)\right|>2 \epsilon\right\} \\
& +\mathbb{P}\left\{\left|\sum_{i=1}^{n} \omega_{i}^{*}\left(t_{0}, h\right)\left(\left[\frac{1}{m} \sum_{l=1}^{m}\left(y_{i l j}-y_{i l k}\right)^{2}-2\left\{1-\rho_{j k}\left(t_{i}\right)\right\}\right]\right)\right|>2 \epsilon\right\} \\
:= & \left.P_{1} \mid+P_{2}, B_{\text {Blostalistics }}\right\}
\end{aligned}
$$


where $\omega_{i}^{*}\left(t_{0}, h\right):=\omega_{i}\left(t_{0}, h\right) \sqrt{\Sigma_{j j}\left(t_{i}\right) \Sigma_{k k}\left(t_{i}\right)}$.

Let $\boldsymbol{Z}:=\left(\boldsymbol{Z}_{1}^{T}, \ldots, \boldsymbol{Z}_{n}^{T}\right)^{T} \in \mathbb{R}^{n m}$, where $\boldsymbol{Z}_{i}:=\left(y_{i 1 j}+y_{i 1 k}, y_{i 2 j}+y_{i 2 k}, \ldots, y_{i m j}+y_{i m k}\right)^{T}$. We have $\boldsymbol{Z}_{l_{1}}$ is independent of $\boldsymbol{Z}_{l_{2}}$ for any $l_{1} \neq l_{2}$. Let

$$
\mathbf{B}:=\left(\begin{array}{cccc}
\sqrt{\omega_{1}^{*}\left(t_{0}, h\right)} \cdot \mathbf{I}_{m} & 0 & \cdots & 0 \\
0 & \sqrt{\omega_{2}^{*}\left(t_{0}, h\right)} \cdot \mathbf{I}_{m} & & 0 \\
& & \ddots & \\
0 & 0 & & \sqrt{\omega_{n}^{*}\left(t_{0}, h\right)} \cdot \mathbf{I}_{m}
\end{array}\right)
$$

be a $m n$ by $m n$ diagonal matrix. Then we can rewrite $P_{1}$ as $P_{1}=\mathbb{P}\left(\left|\|\mathbf{B} \boldsymbol{Z}\|_{2}^{2}-E\|\mathbf{B} \boldsymbol{Z}\|_{2}^{2}\right|>\right.$ $2 m \epsilon)$. Using the property of Gaussian distribution, we have $\mathbf{B} Z \sim N_{m n}(\mathbf{0}, \mathbf{Q})$, where $\mathbf{Q}:=\mathbf{B} \operatorname{cov}(\boldsymbol{Z}) \mathbf{B}$ and

$$
\operatorname{cov}(\boldsymbol{Z})=\left(\begin{array}{cccc}
\operatorname{cov}\left(\boldsymbol{Z}_{1}\right) & 0 & \cdots & 0 \\
0 & \operatorname{cov}\left(\boldsymbol{Z}_{2}\right) & & 0 \\
& & \ddots & \\
0 & 0 & & \operatorname{cov}\left(\boldsymbol{Z}_{n}\right)
\end{array}\right) .
$$

Let $\left\{\operatorname{cov}\left(\boldsymbol{Z}_{i}\right)\right\}_{p q}$ be the $(p, q)$ element of $\operatorname{cov}\left(\boldsymbol{Z}_{i}\right)$. We have

$$
\begin{aligned}
\left|\left\{\operatorname{cov}\left(\boldsymbol{Z}_{i}\right)\right\}_{p q}\right| & =\left|\operatorname{cov}\left(y_{i p j}+y_{i p k}, y_{i q j}+y_{i q k}\right)\right| \\
& =\left|\operatorname{cov}\left(y_{i p j}, y_{i q j}\right)+\operatorname{cov}\left(y_{i p j}, y_{i q k}\right)+\operatorname{cov}\left(y_{i p k}, y_{i q j}\right)+\operatorname{cov}\left(y_{i p k}, y_{i q k}\right)\right| \\
& \leq \frac{\left|\operatorname{cov}\left(x_{i p j}, x_{i q j}\right)+\operatorname{cov}\left(x_{i p j}, x_{i q k}\right)+\operatorname{cov}\left(x_{i p k}, x_{i q j}\right)+\operatorname{cov}\left(x_{i p k}, x_{i q k}\right)\right|}{\min _{r} \Sigma_{r r}\left(t_{i}\right)} \\
& \leq \frac{4\left\|\mathbf{A}\left(t_{i}\right)\right\|_{2}^{|p-q|}\left\|\boldsymbol{\Sigma}\left(t_{i}\right)\right\|_{2}}{\min _{r} \Sigma_{r r}\left(t_{i}\right)} .
\end{aligned}
$$

The last inequality is by using the property of the lag 1 vector autoregressive models. Thus

$$
\begin{aligned}
\|\mathbf{Q}\|_{2} & \leq \max _{1 \leq s \leq m n} \sum_{r=1}^{m n}\left|\mathbf{Q}_{s r}\right| \\
& =\max _{i=1, \ldots, n ; p=1, \ldots, m} \sum_{q=1}^{m} \omega_{i}^{*}\left(t_{0}, h\right)\left|\left\{\operatorname{cov}\left(\boldsymbol{Z}_{i}\right)\right\}_{p q}\right| \\
& \leq \max _{i=1, \ldots, n} \omega_{i}^{*}\left(t_{0}, h\right) \frac{4\left\|\boldsymbol{\Sigma}\left(t_{i}\right)\right\|_{2}}{\min _{r} \Sigma_{r r}\left(t_{i}\right)} \cdot 2 \sum_{q=0}^{\infty}\left\|\mathbf{A}\left(t_{i}\right)\right\|_{2}^{q} \\
& \leq \frac{16 C_{1}}{n h} \cdot \frac{\xi \sup _{t \in[0,1]}\|\mathbf{\Sigma}(t)\|_{2}}{1-\sup _{t \in[0,1]}\|\mathbf{A}(t)\|_{2}} .
\end{aligned}
$$


The last inequality is due to the fact that $\omega_{i}^{*}\left(t_{0}, h\right)=\omega_{i}\left(t_{0}, h\right) \sqrt{\Sigma_{j j}\left(t_{i}\right) \Sigma_{k k}\left(t_{i}\right)} \leq \frac{2}{n h}$. $\sup _{v} K(v) \cdot \sup _{t} \max _{r} \Sigma_{r r}(t)$.

Finally, using Lemma I.2 in Negahban and Wainwright (2011), we have

$$
\begin{aligned}
\mathbb{P}\left(\left|\|\mathbf{B} \boldsymbol{Z}\|_{2}^{2}-\mathbb{E}\|\mathbf{B} \boldsymbol{Z}\|_{2}^{2}\right|>2 m \epsilon\right) & \leq 2 \exp \left\{-\frac{m n}{2}\left(\frac{\epsilon}{2 n\|\mathbf{Q}\|_{2}}-\frac{2}{\sqrt{m n}}\right)^{2}\right\}+2 \exp \left(-\frac{m n}{2}\right) \\
& \leq 4 \exp \left\{-\frac{m n}{2}\left(\frac{\epsilon}{4 n\|\mathbf{Q}\|_{2}}\right)^{2}\right\}
\end{aligned}
$$

for large enough $n$.

Using the same technique, we can show that $P_{2}$ in Equation (A.5) can also be controlled by the bound in (A.7). So using the union bound, we have

$$
\begin{aligned}
\mathbb{P}\left[\max _{j, k}\left|\left\{\mathbf{S}\left(t_{0}\right)\right\}_{j k}-\mathbb{E}\left\{\mathbf{S}\left(t_{0}\right)\right\}_{j k}\right|>\epsilon\right] & \leq \sum_{j, k} \mathbb{P}\left[\left|\left\{\mathbf{S}\left(t_{0}\right)\right\}_{j k}-\mathbb{E}\left\{\mathbf{S}\left(t_{0}\right)\right\}_{j k}\right|>\epsilon\right] \\
& \leq 8 d^{2} \exp \left(-\frac{m \epsilon^{2}}{32 n\|\mathbf{Q}\|_{2}^{2}}\right) .
\end{aligned}
$$

Thus, using Equations (A.6) and (A.8), we have

$$
\begin{aligned}
\max _{j, k}\left|\left\{\mathbf{S}\left(t_{0}\right)\right\}_{j k}-\mathbb{E}\left\{\mathbf{S}\left(t_{0}\right)\right\}_{j k}\right| & =O_{P}\left(\|\mathbf{Q}\|_{2} \sqrt{\frac{n \log d}{m}}\right) \\
& =O_{P}\left[\frac{\xi \cdot \sup _{t \in[0,1]}\|\mathbf{\Sigma}(t)\|_{2}}{h\left\{1-\sup _{t \in[0,1]}\|\mathbf{A}(t)\|_{2}\right\}} \sqrt{\frac{\log d}{m n}}\right] .
\end{aligned}
$$

This completes the proof.

Proof of Lemma 3.1. The rate of convergence in Lemma 3.1 can be obtained by balancing the convergence rates in Lemmas A.1 and A.2. More specifically, we first have

$$
\left\|\mathbf{S}\left(t_{0}\right)-\Sigma\left(t_{0}\right)\right\|_{\max } \leq\left\|\mathbf{S}\left(t_{0}\right)-\mathbb{E} \mathbf{S}\left(t_{0}\right)\right\|_{\max }+\left\|\mathbb{E} \mathbf{S}\left(t_{0}\right)-\Sigma\left(t_{0}\right)\right\|_{\max } .
$$

For notational simplicity, we denote $\theta:=\xi \sup _{t \in[0,1]}\|\boldsymbol{\Sigma}(t)\|_{2} /\left\{1-\sup _{t \in[0,1]}\|\mathbf{A}(t)\|_{2}\right\}$. We then have

$$
\left\|\mathbf{S}\left(t_{0}\right)-\boldsymbol{\Sigma}\left(t_{0}\right)\right\|_{\max }=O_{P}\left(h+\frac{1}{n^{2} h^{1+\eta}}+\frac{\theta}{h} \sqrt{\frac{\log d}{m n}}\right) .
$$

We first balance the first and third terms in the above upper bound, having that

$$
h=\frac{\theta}{h} \sqrt{\frac{\log d}{m n}} \Rightarrow h=\left(\theta \sqrt{\frac{\log d}{m n}}\right)^{1 / 2} .
$$


We then balance the first and second terms, and have that

$$
h=\frac{1}{n^{2} h^{1+\eta}} \Rightarrow h=n^{-\frac{2}{2+\eta}} .
$$

Based on the above two results, we have that, on one hand, if $\left(\theta \sqrt{\frac{\log d}{m n}}\right)^{1 / 2}>n^{-\frac{2}{2+\eta}}$, we can set

$$
h=\left(\theta \sqrt{\frac{\log d}{m n}}\right)^{1 / 2}
$$

Then we have

$$
h=\frac{\theta}{h} \sqrt{\frac{\log d}{m n}}>\frac{1}{n^{2} h^{1+\eta}} \Rightarrow\left\|\mathbf{S}\left(t_{0}\right)-\mathbf{\Sigma}\left(t_{0}\right)\right\|_{\max }=O_{P}\left\{\left(\theta \sqrt{\frac{\log d}{m n}}\right)^{1 / 2}\right\} .
$$

On the other hand, if $\left(\theta \sqrt{\frac{\log d}{m n}}\right)^{1 / 2} \leq n^{-\frac{2}{2+\eta}}$, we can set

$$
h=n^{-\frac{2}{2+\eta}}
$$

Then we have

$$
h=\frac{1}{n^{2} h^{1+\eta}} \geq \frac{\theta}{h} \sqrt{\frac{\log d}{m n}} \Rightarrow\left\|\mathbf{S}\left(t_{0}\right)-\Sigma\left(t_{0}\right)\right\|_{\max }=O_{P}\left(n^{-\frac{2}{2+\eta}}\right) .
$$

Combining (A.9) and (A.10), we have the desired result.

\section{B Proof of Lemma 3.2}

To prove Lemma 3.2, we need an improved upper bound on the distance between $\mathbf{S}\left(t_{0}\right)$ and $\mathbb{E} \mathbf{S}\left(t_{0}\right)$. We provide such a result in Lemma B.1. The proof of Lemma B.1 can be regarded as an extension to the proof of Lemma 6 in Zhou et al. (2010).

Lemma B.1. Suppose that Assumptions (B1), (B2), and (B3) in Lemma 3.2 hold, and $n^{-2 / 5}<h<1$. Then we have there exist absolute positive constants $C_{4}$ and $C_{5}$, such that for

$$
\epsilon<\frac{C_{4}\left\{\Sigma_{j j}^{2}\left(t_{0}\right) \Sigma_{k k}^{2}\left(t_{0}\right)+\Sigma_{j k}^{2}\left(t_{0}\right)\right\}}{\max _{i=1, \ldots, n} K\left\{\left(t_{i}-t_{0}\right) / h\right\} \Sigma_{j j}\left(t_{i}\right) \Sigma_{k k}\left(t_{i}\right)}
$$

we have

$$
\mathbb{P}\left[\left|\left\{\mathbf{S}\left(t_{0}\right)\right\}_{j k}-\mathbb{E}\left\{\mathbf{S}\left(t_{0}\right)\right\}_{j k}\right|>\epsilon\right] \leq 2 \exp \left(-C_{5} m n h \epsilon^{2}\right)
$$


Proof. By the definition of $\mathbf{S}\left(t_{0}\right)$, we have

$$
\begin{aligned}
\mathbb{P}\left[\left|\left\{\mathbf{S}\left(t_{0}\right)\right\}_{j k}-\mathbb{E}\left\{\mathbf{S}\left(t_{0}\right)\right\}_{j k}\right|>\epsilon\right]= & \mathbb{P}\left[\left|\sum_{i=1}^{n} w_{i}\left(t_{0}, h\right)\left\{\frac{1}{m} \sum_{l=1}^{m} x_{i l j} x_{i l k}-\Sigma_{j k}\left(t_{i}\right)\right\}\right|>\epsilon\right] \\
\leq & \mathbb{P}\left[\sum_{i=1}^{n} w_{i}\left(t_{0}, h\right)\left\{\frac{1}{m} \sum_{l=1}^{m} x_{i l j} x_{i l k}-\Sigma_{j k}\left(t_{i}\right)\right\}>\epsilon\right] \\
& +\mathbb{P}\left[\sum_{i=1}^{n} w_{i}\left(t_{0}, h\right)\left\{-\frac{1}{m} \sum_{l=1}^{m} x_{i l j} x_{i l k}+\Sigma_{j k}\left(t_{i}\right)\right\}>\epsilon\right] \\
:= & P_{3}+P_{4} .
\end{aligned}
$$

By Markov's inequality, $\forall r>0$,

$$
\begin{aligned}
P_{3} & =\mathbb{P}\left(\exp \left[m n r \sum_{i=1}^{n} w_{i}\left(t_{0}, h\right)\left\{\frac{1}{m} \sum_{l=1}^{m} x_{i l j} x_{i l k}-\Sigma_{j k}\left(t_{i}\right)\right\}\right]>e^{m n r \epsilon}\right) \\
& \leq \frac{1}{e^{m n r \epsilon}} \mathbb{E} \exp \left[r \sum_{i=1}^{n} \frac{2}{h} K\left(\frac{t_{i}-t_{0}}{h}\right) \sum_{l=1}^{m}\left\{x_{i l j} x_{i l k}-\Sigma_{j k}\left(t_{i}\right)\right\}\right] \\
& =e^{-m n r \epsilon} \prod_{i=1}^{n} \exp \left\{-m r \frac{2}{h} K\left(\frac{t_{i}-t_{0}}{h}\right) \Sigma_{j k}\left(t_{i}\right)\right\} \prod_{i=1}^{n}\left[\mathbb{E} \exp \left\{r \frac{2}{h} K\left(\frac{t_{i}-t_{0}}{h}\right) x_{i l j} x_{i l k}\right\}\right]^{m} .
\end{aligned}
$$

The last equality is due to that $\left\{\boldsymbol{X}^{t_{i}}\right\}_{i=1}^{n}$ are independent and $\left\{\boldsymbol{x}_{i l}\right\}_{l=1}^{m}$ are i.i.d.. Using the same technique, we can get similar result for $P_{4}$. The rest of the proof can be derived by following Lemma 6 in Zhou et al. (2010), where we replace $n$ with $m n$. Here the assumption that $n^{-2 / 5}<h<1$ and Assumption (B2) are required in the proof of Proposition 5 in Zhou et al. (2010).

Using Lemma B.1, we can now proceed to prove Lemma 3.2. Because if the kernel function satisfies Assumption (A2) for some $\eta=\eta_{1}>0$, then this kernel function also satisfies Assumption (A2) for $\eta=\max \left(3, \eta_{1}\right)$, so without loss of generality in the sequel we assume that, in Assumption (A2), $\eta \geq 3$.

Proof of Lemma 3.2. Using Lemma B.1, we have

$$
\begin{aligned}
\mathbb{P}\left[\max _{j k}\left|\left\{\mathbf{S}\left(t_{0}\right)\right\}_{j k}-\mathbb{E}\left\{\mathbf{S}\left(t_{0}\right)\right\}_{j k}\right|>\epsilon\right] & \leq \sum_{j \neq k} \mathbb{P}\left[\left|\left\{\mathbf{S}\left(t_{0}\right)\right\}_{j k}-\mathbb{E}\left\{\mathbf{S}\left(t_{0}\right)\right\}_{j k}\right|>\epsilon\right] \\
& \leq \exp \left(2 \log d-C_{5} m n h \epsilon^{2}\right),
\end{aligned}
$$

for $n^{-2 / 5}<h<1$. Accordingly, we have

$$
\max _{j k}\left|\left\{\mathbf{S}\left(t_{0}\right)\right\}_{j k}-\mathbb{E}\left\{\mathbf{S}\left(t_{0}\right)\right\}_{j k}\right|=O_{P}\left(\sqrt{\frac{\log d}{m n h}}\right)
$$


Together with Lemma A.1, we have

$$
\left\|\mathbf{S}\left(t_{0}\right)-\mathbf{\Sigma}\left(t_{0}\right)\right\|_{\max }=O_{P}\left(h+\frac{1}{n^{2} h^{1+\eta}}+\sqrt{\frac{\log d}{m n h}}\right) .
$$

Similarly as the proof of Lemma 3.1, to balance the first and third terms, we set

$$
h=\sqrt{\frac{\log d}{m n h}} \Rightarrow h=\left(\frac{\log d}{m n}\right)^{1 / 3} .
$$

To balance the first and second terms, we set

$$
h=\frac{1}{n^{2} h^{1+\eta}} \Rightarrow h=\frac{1}{n^{2 /(2+\eta)}} .
$$

If $\left(\frac{\log d}{m n}\right)^{1 / 3}>\frac{1}{n^{2 /(2+\eta)}}$, we set

$$
h=\left(\frac{\log d}{m n}\right)^{1 / 3}
$$

Then we have

$$
h=\sqrt{\frac{\log d}{m n h}}>\frac{1}{n^{2} h^{1+\eta}} \Rightarrow\left\|\mathbf{S}\left(t_{0}\right)-\mathbf{\Sigma}\left(t_{0}\right)\right\|_{\max }=O_{P}\left\{\left(\frac{\log d}{m n}\right)^{1 / 3}\right\} .
$$

Note that $\eta \geq 3$ implies that $h>n^{-2 /(2+\eta)}>n^{-2 / 5}$.

If $\left(\frac{\log d}{m n}\right)^{1 / 3} \leq \frac{1}{n^{2 /(2+\eta)}}$, we set

$$
h=\frac{1}{n^{2 /(2+\eta)}} .
$$

Then we have

$$
h=\frac{1}{n^{2} h^{1+\eta}} \geq \sqrt{\frac{\log d}{m n h}} \Rightarrow\left\|\mathbf{S}\left(t_{0}\right)-\mathbf{\Sigma}\left(t_{0}\right)\right\|_{\max }=O_{P}\left\{\frac{1}{n^{2 /(2+\eta)}}\right\} .
$$

Combining B.1 and B.2 we have the desired result.

\section{References}

Banerjee, O., El Ghaoui, L., and d'Aspremont, A. (2008). Model selection through sparse maximum likelihood estimation for multivariate Gaussian or binary data. The Journal of Machine Learning Research, 9:485-516.

Bartzokis, G., Beckson, M., Lu, P. H., Nuechterlein, K. H., Edwards, N., and Mintz, J. (2001). Age-related changes in frontal and temporal lobe volumes in men: a magnetic resonance imaging study. Archives of General Psychiatry, 58(5):461-465. 
Biswal, B. B., Mennes, M., Zuo, X.-N., Gohel, S., Kelly, C., Smith, S. M., Beckmann, C. F., Adelstein, J. S., Buckner, R. L., Colcombe, S., et al. (2010). Toward discovery science of human brain function. Proceedings of the National Academy of Sciences, 107(10):47344739 .

Blakemore, S.-J. (2012). Imaging brain development: the adolescent brain. Neuroimage, 61(2):397-406.

Braun, U., Plichta, M. M., Esslinger, C., Sauer, C., Haddad, L., Grimm, O., Mier, D., Mohnke, S., Heinz, A., Erk, S., et al. (2012). Test-retest reliability of resting-state connectivity network characteristics using fMRI and graph theoretical measures. Neuroimage, 59(2):1404-1412.

Cai, T., Liu, W., and Luo, X. (2011). A constrained L1 minimization approach to sparse precision matrix estimation. Journal of the American Statistical Association, 106(494):594-607.

Chen, G., Glen, D. R., Saad, Z. S., Paul Hamilton, J., Thomason, M. E., Gotlib, I. H., and Cox, R. W. (2011). Vector autoregression, structural equation modeling, and their synthesis in neuroimaging data analysis. Computers in Biology and Medicine, 41(12):11421155 .

Danaher, P., Wang, P., and Witten, D. M. (2013). The joint graphical lasso for inverse covariance estimation across multiple classes. Journal of the Royal Statistical Society. Series B, Statistical Methodology (in press).

Dempster, A. P. (1972). Covariance selection. Biometrics, 28(1):157-175.

Drton, M. and Perlman, M. D. (2007). Multiple testing and error control in Gaussian graphical model selection. Statistical Science, 22(3):430-449.

Drton, M. and Perlman, M. D. (2008). A SINful approach to Gaussian graphical model selection. Journal of Statistical Planning and Inference, 138(4):1179-1200.

Eloyan, A., Muschelli, J., Nebel, M. B., Liu, H., Han, F., Zhao, T., Barber, A. D., Joel, S., Pekar, J. J., Mostofsky, S. H., et al. (2012). Automated diagnoses of attention deficit hyperactive disorder using magnetic resonance imaging. Frontiers in Systems Neuroscience, 6(61):1-9.

Friedman, J., Hastie, T., and Tibshirani, R. (2008). Sparse inverse covariance estimation with the graphical lasso. Biostatistics, 9(3):432-441. 
Friston, K. (2011). Functional and effective connectivity: a review. Brain Connectivity, $1(1): 13-36$.

Giedd, J. N., Blumenthal, J., Jeffries, N. O., Castellanos, F. X., Liu, H., Zijdenbos, A., Paus, T., Evans, A. C., and Rapoport, J. L. (1999). Brain development during childhood and adolescence: a longitudinal MRI study. Nature Neuroscience, 2(10):861-863.

Guo, J., Levina, E., Michailidis, G., and Zhu, J. (2011). Joint estimation of multiple graphical models. Biometrika, 98(1):1-15.

Han, F. and Liu, H. (2013). Transition matrix estimation in high dimensional time series. In Proceedings of the 30th International Conference on Machine Learning, pages 172-180.

Harrison, L., Penny, W. D., and Friston, K. (2003). Multivariate autoregressive modeling of fMRI time series. Neuroimage, 19(4):1477-1491.

Kolar, M., Song, L., Ahmed, A., and Xing, E. P. (2010). Estimating time-varying networks. The Annals of Applied Statistics, 4(1):94-123.

Kolar, M. and Xing, E. P. (2009). Sparsistent estimation of time-varying discrete markov random fields. arXiv preprint arXiv:090\%.233\%.

Lauritzen, S. L. (1996). Graphical Models, volume 17. Oxford University Press.

Liu, W. and Luo, X. (2012). High-dimensional sparse precision matrix estimation via sparse column inverse operator. arXiv preprint arXiv:1203.3896.

Loh, P.-L. and Wainwright, M. J. (2012). High-dimensional regression with noisy and missing data: Provable guarantees with nonconvexity. The Annals of Statistics, 40(3):16371664 .

Meinshausen, N. and Bühlmann, P. (2006). High-dimensional graphs and variable selection with the lasso. The Annals of Statistics, 34(3):1436-1462.

Negahban, S. and Wainwright, M. J. (2011). Estimation of (near) low-rank matrices with noise and high-dimensional scaling. The Annals of Statistics, 39(2):1069-1097.

Pang, H., Liu, H., and Vanderbei, R. (2013). The fastclime package for linear programming and constrained L1-minimization approach to sparse precision matrix estimation in $\mathrm{R}$. CRAN. 
Penny, W., Ghahramani, Z., and Friston, K. (2005). Bilinear dynamical systems. Philosophical Transactions of the Royal Society B: Biological Sciences, 360(1457):983-993.

Power, J. D., Cohen, A. L., Nelson, S. M., Wig, G. S., Barnes, K. A., Church, J. A., Vogel, A. C., Laumann, T. O., Miezin, F. M., Schlaggar, B. L., et al. (2011). Functional network organization of the human brain. Neuron, 72(4):665-678.

Rogers, B. P., Katwal, S. B., Morgan, V. L., Asplund, C. L., and Gore, J. C. (2010). Functional MRI and multivariate autoregressive models. Magnetic Resonance Imaging, 28(8):1058-1065.

Rothman, A. J., Bickel, P. J., Levina, E., and Zhu, J. (2008). Sparse permutation invariant covariance estimation. Electronic Journal of Statistics, 2:494-515.

Shaw, P., Kabani, N. J., Lerch, J. P., Eckstrand, K., Lenroot, R., Gogtay, N., Greenstein, D., Clasen, L., Evans, A., Rapoport, J. L., et al. (2008). Neurodevelopmental trajectories of the human cerebral cortex. The Journal of Neuroscience, 28(14):3586-3594.

Song, L., Kolar, M., and Xing, E. P. (2009a). KELLER: estimating time-varying interactions between genes. Bioinformatics, 25(12):i128-i136.

Song, L., Kolar, M., and Xing, E. P. (2009b). Time-varying dynamic bayesian networks. Advances in Neural Information Processing Systems, 22:1732-1740.

Tasaki, H. (2009). Convergence rates of approximate sums of Riemann integrals. Journal of Approximation Theory, 161(2):477-490.

Valdés-Sosa, P. A., Sánchez-Bornot, J. M., Lage-Castellanos, A., Vega-Hernández, M., Bosch-Bayard, J., Melie-García, L., and Canales-Rodríguez, E. (2005). Estimating brain functional connectivity with sparse multivariate autoregression. Philosophical Transactions of the Royal Society B: Biological Sciences, 360(1457):969-981.

Van Dijk, K. R., Sabuncu, M. R., and Buckner, R. L. (2012). The influence of head motion on intrinsic functional connectivity MRI. Neuroimage, 59(1):431-438.

Wang, Z., Han, F., and Liu, H. (2013). Sparse principal component analysis for high dimensional multivariate time series. Journal of Machine Learning Research (AISTATS Track), pages $48-56$.

Yuan, M. (2010). High dimensional inverse covariance matrix estimation via linear programming. The Journal of Machine Learning Research, 11:2261-2286. 
Yuan, M. and Lin, Y. (2007). Model selection and estimation in the Gaussian graphical model. Biometrika, 94(1):19-35.

Zhao, T., Liu, H., Roeder, K., Lafferty, J., and Wasserman, L. (2012). The huge package for high-dimensional undirected graph estimation in $\mathrm{R}$. The Journal of Machine Learning Research, 13:1059-1062.

Zhou, S., Lafferty, J., and Wasserman, L. (2010). Time varying undirected graphs. Machine Learning, 80(2-3):295-319. 